\title{
Computer-Generated Keys to the Flora of Egypt. 8. The Lamiaceae
}

\author{
A. El-Gazzar ${ }^{(1) \#}$, A. El-Ghamery ${ }^{(2)}$, A.H. Khattab ${ }^{(3)}$, B.S. El-Saeid ${ }^{(2)}$,A.A. El-Kady ${ }^{(2)}$ \\ (1)Botany and Microbiology Department, Faculty of Science, El-Arish University, El- \\ Arish, N. Sinai, Egypt; ${ }^{(2)}$ Botany and Microbiology Department, Faculty of Science, \\ Al-Azhar University, Cairo, Egypt; ${ }^{(3)}$ The Herbarium, Botany Department, Faculty of \\ Science, Cairo University, Cairo, Egypt.
}

\begin{abstract}
$\mathbf{M}$ ANUALLY-constructed keys to many groups of the Egyptian flora are in urgent need of improvement and updating. To construct a conventional substitute of the key to representatives of the Lamiaceae, a data matrix was compiled to include 48 characters recorded for each of the 52 species (with three subspecies and one variety) belonging to 24 genera which represent this family in the flora of Egypt. The 48 characters were accurately defined to cover as much of the easily observable aspects of vegetative and floral variation in the plants as possible. The data matrix was analyzed using the key-generating package of programs DELTA. The analysis produced a conventional key with a detailed description of every species in terms of the 48 characters. The key is decidedly a marked improvement over its predecessors in that it is strictly comparative. Updating the nomenclature of the plants led to the first recording of the genus Thymbra in the flora of Egypt.
\end{abstract}

Keywords: Conventional key, DELTA, Egypt, Flora, Lamiaceae, Thymbra.

\section{Introduction}

The Lamiaceae Lindl. (nom.altern. Labiatae Juss.) is a relatively large family with $240-255$ genera encompassing 6900-7200 species (Bergianska, 2014 and Chase et al., 2016). The plants are predominantly annual or perennial herbs, but may be shrubs or small trees (e.g., most Hyptis spp.). The stem is usually erect but may be prostrate or rhizomatous (e.g., some Mentha spp.); young stems are quadrangular in cross-section; pith solid or hollow; nodes are consistently unilacunar with 1-2 traces per leaf gap. The density of indumentum on vegetative organs ranges from entirely glabrous to thickly pilose or white-tomentose. Epidermal trichomes frequently found in various combinations on vegetative and floral parts are uni- or multicellular, glandular or eglandular, simple, branched, stellate, or 'dendroid'. The leaves are usually opposite decussate, whorled only in Dysophylla (El-Gazzar \& Watson, 1967), consistently ex-stipulate, petiolate or sessile, simple to variously dissected, flat or ericoid with recurved margins as in some Lavandula spp. and Rosmarinus officinalis (El-Gazzar \& Watson, 1970). The inflorescence is a terminal spike, a globose aggregate of cymes or a lax-dense verticillasters in acropetal succession where the number of flowers per bract axil varies from 1 to many. Bracts are usually broad and leafy; bracteols are often absent. The flower is hermaphrodite, pentamerous, hypogynous and strongly zygomorphic. Calyx of 5 united persistent sepals, usually 2-lipped, with 2-5 teeth, or with 10 teeth in some Leucas and Marrubium species; teeth are rarely equal and the calyx is regular (e.g., in Mentha). Corolla of 5 united petals, often showy, 2-lipped, configuration is often characteristic of most genera; upper lip of two lobes, commonly vaulted (e.g., Lamium, Salvia), glabrous or with a thick tuft of hairs on its outer surface (e.g., in Lamium, Phlomis), but may be short or absent (e.g., in Teucrium and Ajuga). Lower lip consists of 3 unequal and variously shaped lobes. Stamens 2 or 4 , epipetalous, the posterior stamen is usually absent or present as a minute staminode (e.g., in Rosmarinus), exserted or included in corolla; filaments hairy or glabrous, straight or curved and included in the vault of the upper or lower corolla lip; anthers usually dithecous or monothecous, thecae divaricated or parallel. In Salvia, filaments of the two stamens are short and the two lobes of each anther are widely separated by a long filiform connective which forms a lever-like structure

"Corresponding author email: elgazzar_adel@hotmail.com

DOI: 10.21608/ejbo.2018.4861.1201

Edited by: Prof. Dr. Monier M. Abd El-Ghani, Faculty of Science, Cairo University, Cairo, Egypt.

(C)2019 National Information and Documentation Center (NIDOC) 
with the staminal filament thus giving the stamen a high degree of versatility suited for pollination by insects (El-Gazzar et al., 1968 and Walker \& Sytsma, 2007). Gynoecium is 2-carpellate, usually 4-locular by intrusion of carpel wall forming 'false septum'. Ovary is often 4-locular with 1 ovule per locule, glabrous or hairy, with a gynobasic style, or entire and 4-ovuled (e.g., in Ajuga, Teucrium) with a terminal style. Ovary usually on a fleshy, entire, irregularly or often 4-lobed nectariferous disc, usually anterior lobe is longer than others. Style is glabrous or hairy, usually with 2 equal or unequal stigmatic arms, sometimes entire with 1 stigma-lobe vestigial, or stigma capitate or very rarely 4-lobed. A 4-loculed ovary develops into four 1-seeded mericarps (nutlets), sometimes fewer by abortion; mericarps often with sculptured, tuberculate, hairy or rarely winged pericarp, mucilage cells often present. An entire ovary develops into a drupe.

The Lamiaceae are cosmopolitan but with a marked absence in areas with extremely harsh environmental conditions in the Arctic Circle, the African Sahara, central Arabia and the expanses of the Mongolian desert. Most genera and species show a marked preference for the temperate environment (Watson \& Dallwitz, 1992 onwards).

Numerous members of Lamiaceae are rich in strongly aromatic essential oils in their shoot system (Guenther, 1974 and Hegnauer, 1966), while others have copious amounts of fixed oils in their seeds (Hagemann et al., 1967). Species of many genera (e.g., Lavandula, Mentha, Origanum) derive their economic importance from their production of essential oils which are the main constituents of perfumes, toothpastes and food and drink flavoring additives. Species of other genera (e.g., Rosmarinus officinalis, Salvia officinalis) are commonly used as culinary herbs. Essential oils of many species have for centuries been used worldwide to treat various ailments (Balali et al., 2012 and Mikaili et al., 2013), as antioxidants (Hussain et al., 2011 and Delnavazi et al., 2014), antibacterial (Stefanakis et al., 2013), antifungal (Medjahed et al., 2016), insecticides (Mossa, 2016) and several medicinal applications (e.g., Caldas et al., 2014). A few herbaceous species (e.g., Salvia splendens, Salvia coccinea, Molucella laevis) are grown as garden ornamentals.

The Lamiaceae are represented in the flora of Egypt by 23 genera and 54 species (Boulos, 2002), including Clerodendrum acerbianum which traditionally belonged in the Verbenaceae but was among the taxa transferred to Lamiaceae by Cantino (1992). Manually constructed keys to these taxa were provided by Muschler (1912), Montasir \& Hassib (1956), Täckholm (1956, 1974), El-Husseini (1986) and Boulos (2002). The same as all manually constructed keys, those of the Lamiaceae (and several other families with relatively large representation in the local flora) are in urgent need for updating and improvement. Hence, the ongoing work on benefiting from the inherent versatility of the package of keygenerating computer programs DELTA in constructing much improved identification keys to these groups. Previous studies in this series dealt with the Leguminosae (Mimosoideae and Caesalpinioideae), Chenopodiaceae, Pantaginaceae, Solanaceae, LeguminosaePapilionoideae, Boraginaceae and Acanthaceae s.l. (El-Gazzar et al., 2008 a, b; 2009 a, b; 2012 and $2015 \mathrm{a}, \mathrm{b}$, respectively).

\section{Materials and Methods}

Specimens of 52 species (with three subspecies and one variety) belonging to 24 genera of the Lamiaceae were obtained from the herbarium of the Faculty of Science, Cairo University. Identification of all taxa was accurately scrutinized according to El-Gazzar (1969) and El-Husseini (1986), and updated according to the two major nomenclatural databases [http://www.theplantlist. org/] and [http://www.tropicos.org/]".

Available specimens of the 54 taxa (52 species, three subspecies and one variety) were examined in detail for any easily observable and seemingly stable aspects of variation in vegetative and floral morphology. The 48 characters which met these two basic criteria were recorded comparatively for every species in a data matrix which was subjected to analysis under the program suit DELTA (Dallwitz et al., 2000) to generate a conventional identification key and detailed description of every taxon in terms of the recorded characters.

\section{$\underline{\text { Results }}$}

\section{The characters}

The 48 characters recorded comparatively for the 54 taxa belonging to 24 genera representing the Lamiaceae in the flora of Egypt are listed in 
Table 1. The numbers of qualitative, multistate and quantitative characters in that list are 38, 7 and 3 , respectively. The wide range of variation in leaf morphology provided a wealth of potentially useful characters. Thus, leaf blades may be simple (Figs. 1-7), 3-lobed (Fig. 8), partially pinnate (Fig. 9), pinnate (Fig. 10) or bi- to tri-pinnate with filiform to broad pinnae and pinnules (Fig. 11). The following shapes of leaf blades were observed:

1- Linear-lanceolate, the blade is at least 6 times as long as broad (Fig. 1),

2- Oblong-ovate, with acute or obtuse apex and rotund to cuneate base (Figs. 2-4),

3- Cordate, similar to the preceding but with deep base (Fig. 5), and

4- Fan-shaped (Fig. 7).

The leaves are sessile-subsessile (Figs. 1, 6) or conspicuously petioled (Figs. 2-5, 7-11), uninerved (Fig. 1), or with palmate (Figs. 7 and 8 ) or pinnate venation (Fig. 5). The leaf margin ranges from entire (Figs. 1, 3, 6) to finely or coarsely denticulate-serrate (Figs. 2, 4), or irregularly sinuate (Fig. 5). Leaf surface is either smooth (Figs. 3, 4), white tomentose (Fig. 7) or rugose (Figs. 2, 5).

TABLE 1. List of the 48 characters and character-states recorded comparatively for 24 genera and 54 taxa representing the Lamiaceae in the flora of Egypt and used to construct a conventional key to them [*= Multistate character; **= Quantitative character $]$.

\#1. Plant/1. herbaceous/2. woody/

\#2. Stem/1. hairy/2. glabrous/

\#3. Stem/1. square/2. terete/

\#4. Stem/1. erect/2. prostrate/

\#5. Stolons/1. present/2. absent/

\#6. Leaves/1. petioled/2. sessile-subsessile/

\#7. Leaves/1. simple/2. 3-lobed/3. partially pinnate/4. uni- to bi-pinnate/*

\#8. Leaf blade/1. linear-lanceolate/2. oblong-ovate/3. cordate/4. orbicular/5. fan-shaped/*

\#9. Leaf blade/cm long/**

$\# 10$. Leaf blade/cm broad/**

\#11. Leaf veins/1. prominent/2. not prominent/

\#12. Leaf veins/1. black/2. not black/

\#13. Leaf margin/1. entire/2. denticulate-serrate/3. sinuate/*

\#14. Leaf margin/1. revolute/2. flat/

\#15. Leaf apex/1. acute/2. obtuse/

\#16. Leaf surface/1. smooth/2. rugose/

\#17. Leaves/1. white-tomentose/2. not white-tomentose/

\#18. Leaf/1. uninerved/2. venation palmate/3. venation pinnate/*

$\# 19$. Inflorescence/1. lax verticillasters/2. dense spike-like/3. terminal globose aggregate/*

\#20. Flowers/1. pedicelled/2. sessile/

\#21. Calyx/1. bilabiate/2. nearly regular/

\#22. Calyx/1. funnel-shaped/2. not funnel-shaped/

\#23. Calyx veins/1. prominent/2. not prominent/

\#24. Calyx teeth/1. 5 or less/2. 8-10/

\#25. Calyx teeth/1. hooked/2. not hooked/

\#26. Calyx teeth/1. spiny-tipped/2. not spiny-tipped/

\#27. Posterior sepal/mm long/**

\#28. Veins in calyx tube/1. 5/2. 10/3. 13/4. 15/*

\#29. Outer surface of petal lobes/1. hairy/2. glabrous/

\#30. Upper lip of corolla/1. vaulted/2. flat-deflexed/3. nearly equal to lower lip/4. reduced/*

\#31. Corolla tube/1. included in calyx/2. much longer than calyx/

\#32. Stamens/1. 2/2. 4/

\#33. Stamens/1. exserted/2. included/ 
TABLE 1. Cont.

\#34. Staminal filaments/1. hairy/2. glabrous/

\#35. Filament-connective attachment/1. lever-like/2. not lever-like/

\#36. Ovary/1. hairy/2. glabrous/

\#37. Style/1. hairy/2. glabrous/

\#38. Style/1. terminal/2. gynobasic/

\#39. Stigma/1. capitate/2. 2 elongate arms/

\#40. Stigmatic lobes/1. equal/2. unequal/

\#41. Fruit/1. 4 nutlets/2. drupe/

\#42. Eglandular hairs on leaves/1. present/2. absent/

\#43. Sessile glandular hairs on leaves/1. present/2. absent/

\#44. Stalked glandular hairs on leaves/1. present/2. absent/

\#45. Branched hairs on leaves/1. present/2. absent/

\#46. Sessile glandular hairs on calyx/1. present/2. absent/

\#47. Stalked glandular hairs on calyx/1. present/2. absent/

\#48. Branched hairs on calyx/1. present/2. absent/
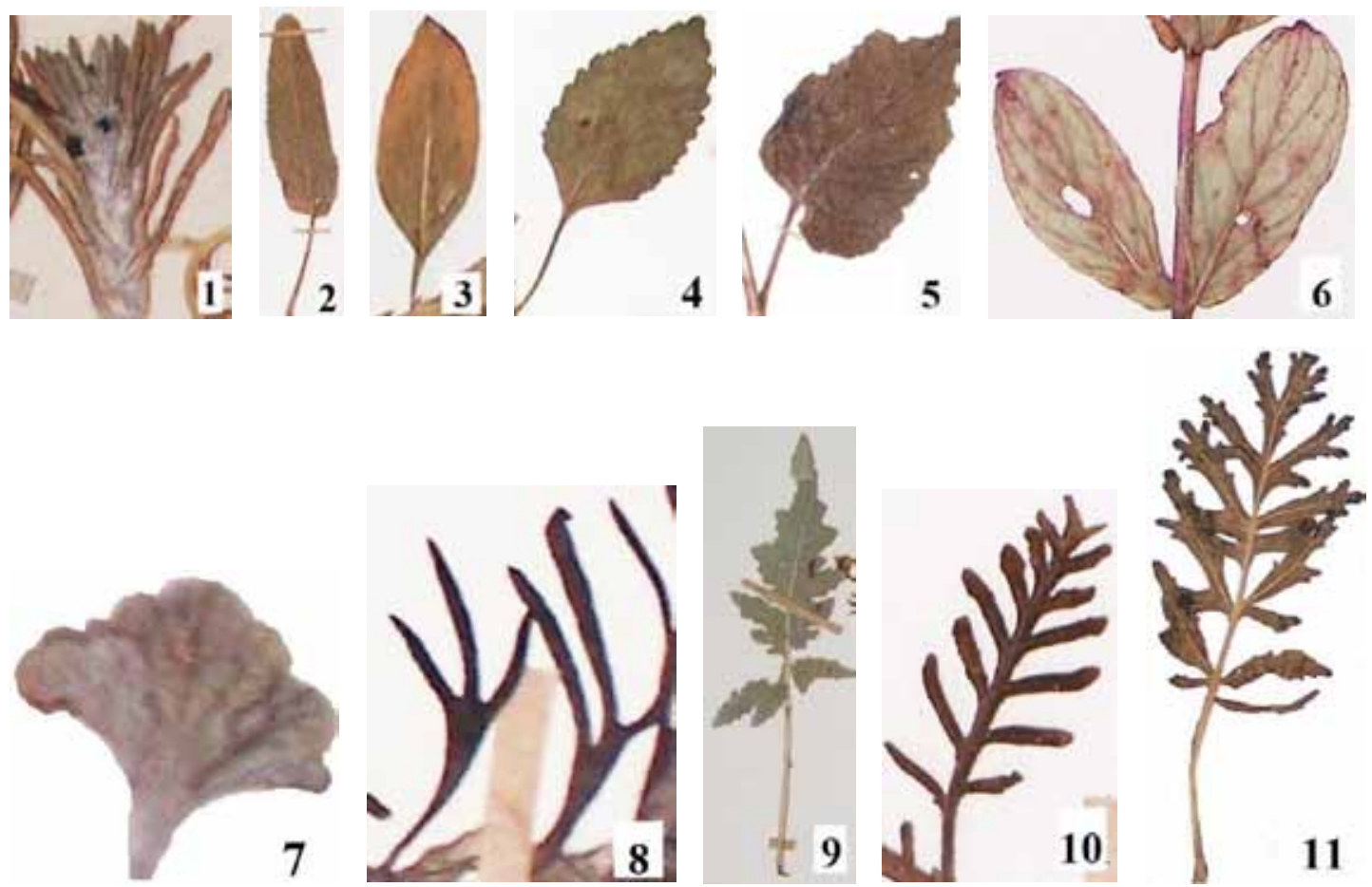

Figs. 1-11. Images illustrating the variation in leaf morphology of Lamiaceae. 1. Ajuga iva: Linear, uninerved; 2. Phlomis floccosa: Oblong with rotund base; 3. Ziziphora capitata: Oblong with cuneate base; 4. Otostegia fruticosa: Ovate; 5. Salvia spinosa: Ovate with cordate base; 6. Mentha longifolia: Sessile, entire margin; 7. Marrubium alysson: Fan-shaped, white-tomentose, palmate venation, veins black; 8. Ajuga chamaepytis: 3-lobed; 9. Salvia palaestina: Partially pinnate; 10. Lavandula canariensis: Pinnate; 11. Eremostachys laciniata: Tri-pinnate.

The DELTA package produces conventional keys and detailed descriptions of the taxa in natural language. When those keys and descriptions become in need of updating by adding more taxa and/or characters, the original data matrix will be required; copies of the list of characters and the data matrix are available on request from A.A. El-Kady (alaa.elkady885@yahoo.com) or B.S. El-Saeid (baraa_elsaied@yahoo.com). 


\section{The bracketed Key}

Characters: 48 indata, 45 included, 37 in key.

Items: 54 indata, 54 included, 54 in key.

Parameters: Rbase $=1.40$ Abase $=2.00$ Reuse $=1.01$ Varywt $=0.80$

Characters included: 1-8 11-26 28-48

Character reliabilities: $1-48,5$

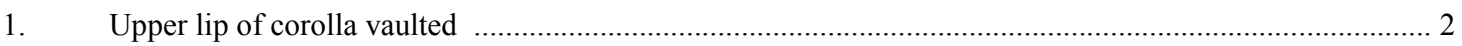

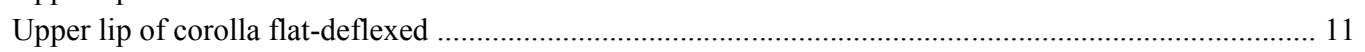

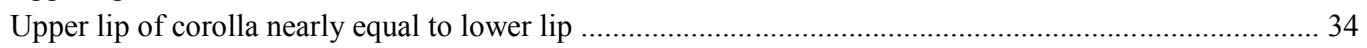

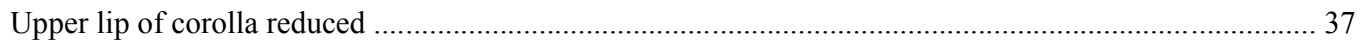

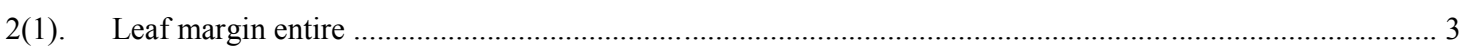

Leaf margin denticulate-serrate ....................................................................................................... 4

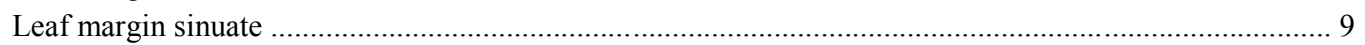

3(2). Leaf veins prominent; Leaf surface rugose; Leaves not white-tomentose;

Calyx nearly regular.

Phlomis aurea Decne.

Leaf veins not prominent; Leaf surface smooth; Leaves white-tomentose;

Calyx bilabiate

Salvia multicaulis Vahl

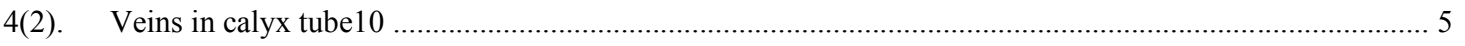

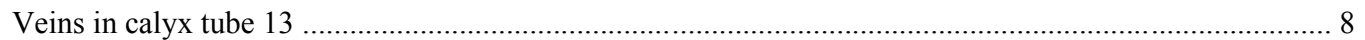

Veins in calyx tube 15 ...................................................... Phlomoides laciniata (L.) Kamelin \& Makhm.

5(4). Leaf apex acute; Leaf venation pinnate; Stamens 2; Filament-connective attachment lever-like ................6 6

Leaf apex obtuse; Leaf venation palmate; Stamens 4; Filament-connective attachment not lever-like ....... 7

6(5). Leaf veins prominent; Leaf blade oblong-ovate; Leaves white-tomentose;

Flowers sessile

Salvia dominica $\mathbf{L}$.

Leaf veins not prominent; Leaf blade cordate; Leaves not white-tomentose;

Flowers pedicelled

Salvia spinosa $\mathbf{L}$.

7(5). Leaf surface smooth; Leaves white-tomentose; Calyx bilabiate;

Calyx teeth 5 or less

Otostigia fruticosa (Forssk.) Schweinf. ex Penzig ssp. fruticosa

Leaf surface rugose; Leaves not white-tomentose; Calyx nearly regular;

Calyx teeth 8-10

Phlomis floccosa D. Don

8(4). Leaf veins prominent; Leaf apex acute; Leaves partially pinnate; Leaf margin flat ..... Salvia verbenaca L. Leaf veins not prominent; Leaf apex obtuse; Leaves simple;

Leaf margin revolute

Salvia lanigera Poir. in Lam.

9(2). Leaf veins prominent; Veins in calyx tube 10; Leaves simple; Corolla tube included in calyx 10

Leaf veins not prominent; Veins in calyx tube 13; Leaves partially pinnate;

Corolla tube much longer than calyx

Salvia palaestina Benth.

10(9). Leaf apex acute; Plant herbaceous; Leaf surface rugose; Leaves not white-tomentose ....... Salvia sclarea L. Leaf apex obtuse; Plant woody; Leaf surface smooth;

Leaves white-tomentose

Otostegia fruticosa (Forssk.) Penzig ssp. schimperi (Benth.) Sebald.

11(1). Veins in calyx tube5

Origanum syriacum L. ssp. sinaicum (Boiss.) Greuter \& Burdet

Veins in calyx tube 10

Veins in calyx tube 13

Veins in calyx tube 15 


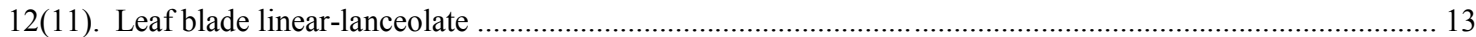

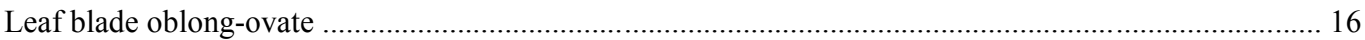

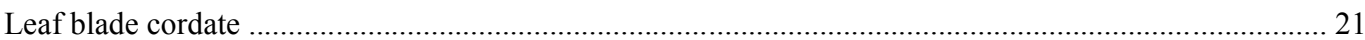

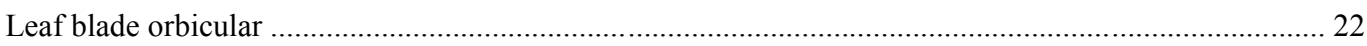

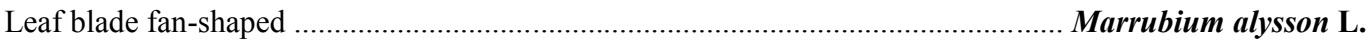

13(12). Leaf margin entire; Inflorescence terminal globose aggregate ............................................................. 14 Leaf margin denticulate-serrate; Inflorescence dense spike-like ................... Leucas neuflizeana Courbon Leaf margin sinuate; Inflorescence lax verticillasters Salvia deserti Decne.

14(13). Leaf apex acute; Stigma capitate; Stigmatic lobes equal; Branched hairs on calyx absent 15 Leaf apex obtuse; Stigma 2 elongate arms; Stigmatic lobes unequal; Branched hairs on calyx present .

Thymbra capitata (L.) Cav.

15(14). Leaves white-tomentose; Leaf uninerved; Calyx nearly regular;

Calyx funnel-shaped

Thymus decussatus Benth.

Leaves not white-tomentose; Leaf venation pinnate; Calyx bilabiate;

Calyx not funnel-shaped

Thymus bovei Benth.

16(12). Leaf veins black

Leaf veins not black

17(16). Leaf venation palmate; Inflorescence lax verticillasters; Calyx not funnel-shaped; Calyx teeth hooked

Marrubium vulgare $\mathbf{L}$. Leaf venation pinnate; Inflorescence dense spike-like; Calyx funnel-shaped; Calyx teeth not hooked ..... 18

18(17). Calyx teeth spiny-tipped; Corolla tube included in calyx; Staminal filaments glabrous; Stigmatic lobes unequal

Ballota saxatilis Sieber ex C. Presl

Calyx teeth not spiny-tipped; Corolla tube much longer than calyx; Staminal filaments hairy; Stigmatic lobes equal

Ballota kaiseri Täckh.

19(16). Leaf veins prominent; Leaf surface rugose; Leaf venation palmate; Inflorescence dense spike-like

Ballota pseudodictamnus (L.) Benth.

Leaf veins not prominent; Leaf surface smooth; Leaf venation pinnate;

Inflorescence lax verticillasters

20(19). Leaf margin entire; Leaf apex acute; Plant woody; Leaves white-tomentose .... Ocimum forsskaolii Benth. Leaf margin denticulate-serrate; Leaf apex obtuse; Plant herbaceous;

Leaves not white-tomentose

Orthosiphon pallidus Royle ex Benth. in Hook.

21(12). Leaf veins prominent; Plant woody; Leaf surface rugose; Inflorescence dense spike-like

Ballota undulata (Sieber ex Fresen.) Benth.

Leaf veins not prominent; Plant herbaceous; Leaf surface smooth; Inflorescence lax verticillasters

Lamium amplexicaule $\mathrm{L}$.

22(12). Leaf margin entire; Leaf apex acute; Stem erect; Leaf surface rugose Leucas inflata Benth. Leaf margin denticulate-serrate; Leaf apex obtuse; Stem prostrate; Leaf surface smooth

Ballota damascena Boiss.

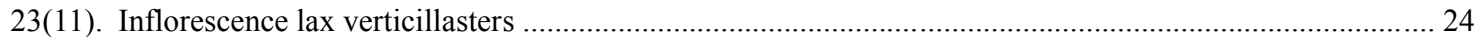

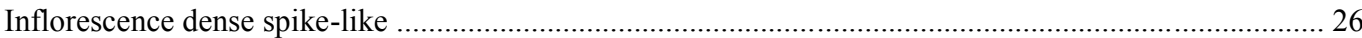

Inflorescence terminal globose aggregate ............................................................... Ziziphora capitata L.

24(23). Leaf veins prominent; Leaves petioled; Flowers pedicelled; Sessile glandular hairs on leaves absent ...... 25 Leaf veins not prominent; Leaves sessile-subsessile; Flowers sessile; 
Sessile glandular hairs on leaves present

Micromeria sinaica Benth.

25(24). Stigmatic lobes equal

Micromeria imbricata (Forssk.) C. Chr.

Stigmatic lobes unequal Micromeria nervosa (Desf.) Benth.

26(23). Leaf veins prominent; Leaves petioled; Leaf blade oblong-ovate; Flowers pedicelled ...... 27

Leaf veins not prominent; Leaves sessile-subsessile; Leaf blade linear-lanceolate;

Flowers sessile

Ziziphora tenuior $\mathbf{L}$.

27(26). Leaf margin entire; Plant woody; Stem hairy;

Leaf margin revolute

Micromeria myrtifolia Boiss. \& Hohen. in Boiss.

Leaf margin denticulate-serrate; Plant herbaceous; Stem glabrous;

Leaf margin flat

Prasium majus L.

28(11). Leaf margin entire

Leaf margin denticulate-serrate

Leaf margin sinuate

Salvia aegyptiaca $\mathrm{L}$.

29(28). Leaf apex acute; Leaf uninerved; Corolla tube much longer than calyx; Staminal filaments glabrous

Lavandula atriplicifolia Benth.

Leaf apex obtuse; Leaf venation pinnate; Corolla tube included in calyx; Staminal filaments hairy. 30

30(29). Plant herbaceous; Leaves petioled; Leaf blade oblong-ovate; Inflorescence dense spike-like

Lallemantia royleana (Benth.) Benth. in DC.

Plant woody; Leaves sessile-subsessile; Leaf blade linear-lanceolate; Inflorescence lax verticillasters Stachys aegyptiaca Pers.

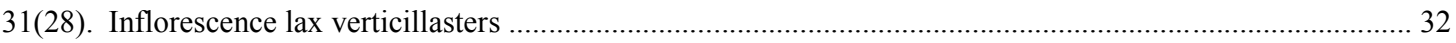

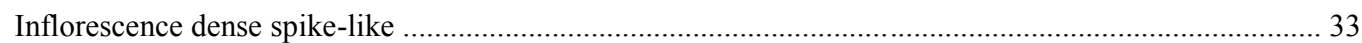

32(31). Leaf apex acute; Leaves uni- to bi-pinnate; Leaf venation pinnate;

Flowers Sessile

Lavandula coronopifolia Poir. in Lam.

Leaf apex obtuse; Leaves simple; Leaf venation palmate;

Flowers pedicelled

Nepeta septemcrenata Ehrenb. ex Benth.

33(31). Leaves petioled; Calyx bilabiate; Calyx veins prominent;

Eglandular hairs on leaves absent

Lavandula pubescens Decne.

Leaves sessile-subsessile; Calyx nearly regular; Calyx veins not prominent;

Eglandular hairs on leaves present

Lavandula canariensis (L.) Mill.

34(1). Leaf veins prominent; Flowers sessile; Eglandular hairs on leaves present ...

Leaf veins not prominent; Flowers pedicelled; Eglandular hairs on leaves absent

35(34). Leaves petioled; Stalked glandular hairs on leaves present

Mentha longifolia (L.) Huds. ssp. typhoides (Briq.) Harley

Leaves sessile-subsessile; Stalked glandular hairs on leaves absent

Mentha longifolia (L.) Huds. var. schimperi (Briq.) Briq.

36(34). Leaf apex acute; Plant woody; Stem hairy; Stem erect

Clerodendrum acerbianum (Vis. ) Benth.

Leaf apex obtuse; Plant herbaceous; Stem glabrous; Stem prostrate Mentha pulegium $\mathrm{L}$.

37(1). Leaf margin entire 38

Leaf margin denticulate-serrate

Teucrium decaisnei C. Presl

Leaf margin sinuate 
38(37). Inflorescence lax verticillasters .... Ajuga chamaepitys (L.) Schreb. ssp. tridactylites (Ging. ex Benth.)

P.H. Davis

Inflorescence dense spike-like Ajuga iva (L.) Schreb.

Inflorescence terminal globose aggregate Teucrium brevifolium Schreb.

39(37). Plant herbaceous; Outer surface of petal lobes glabrous; Stamens included; Branched hairs on leaves present Teucrium polium $\mathbf{L}$.

Plant woody; Outer surface of petal lobes hairy; Stamens exserted;

Branched hairs on leaves absent

Teucrium leucocladum Boiss.

\section{Detailed descriptions}

The detailed descriptions are the sets of characters-states of the 48 characters found in each taxon as recorded in the original data matrix, used in the process of key generation and retrieved from the computer.

Ajuga chamaepitys (L.) Schreb. ssp. tridactylites (Ging. ex Benth.) P.H. Davis Plant woody. Stem hairy. Stem square. Stem prostrate. Stolons absent. Leaves sessile-subsessile. Leaves 3-lobed. Leaf blade linear-lanceolate. Leaf blade $2-2.5 \mathrm{~cm}$ long. Leaf blade $1-1.5 \mathrm{~cm}$ broad. Leaf veins not prominent. Leaf veins not black. Leaf margin entire. Leaf margin flat. Leaf apex acute. Leaf surface smooth. Leaves not white-tomentose. Leaf uninerved. Inflorescence lax verticillasters. Flowers sessile. Calyx nearly regular. Calyx funnel-shaped. Calyx veins not prominent. Calyx teeth 5 or less. Calyx teeth not hooked. Calyx teeth not spiny-tipped. Posterior sepal 2-5 mm long. Veins in calyx tube 10 . Outer surface of petal lobes hairy. Upper lip of corolla reduced. Corolla tube included in calyx. Stamens 4. Stamens exserted. Staminal filaments hairy. Filament-connective attachment not lever-like. Ovary glabrous. Style hairy. Style terminal. Stigma 2 elongate arms. Stigmatic lobes equal. Fruit drupe. Eglandular hairs on leaves present. Sessile glandular hairs on leaves absent. Stalked glandular hairs on leaves absent. Branched hairs on leaves absent. Sessile glandular hairs on calyx absent. Stalked glandular hairs on calyx absent. Branched hairs on calyx absent.

Ajuga iva (L.) Schreb. Plant herbaceous. Stem hairy. Stem square. Stem prostrate. Stolons absent. Leaves sessile-subsessile. Leaves simple. Leaf blade linear-lanceolate. Leaf blade $3-3.5 \mathrm{~cm}$ long. Leaf blade $0.2-0.3 \mathrm{~cm}$ broad. Leaf veins prominent. Leaf veins not black. Leaf margin entire. Leaf margin revolute. Leaf apex obtuse. Leaf surface smooth. Leaves white-tomentose. Leaf uninerved. Inflorescence dense spike-like.
Flowers sessile. Calyx nearly regular. Calyx not funnel-shaped. Calyx veins not prominent. Calyx teeth 5 or less. Calyx teeth not hooked. Calyx teeth not spiny-tipped. Posterior sepal 1-4 mm long. Veins in calyx tube 10. Outer surface of petal lobes hairy. Upper lip of corolla reduced. Corolla tube much longer than calyx. Stamens 4. Stamens exserted. Staminal filaments hairy. Filament-connective attachment not lever-like. Ovary glabrous. Style hairy. Style terminal. Stigma 2 elongate arms. Stigmatic lobes equal. Fruit drupe. Eglandular hairs on leaves present. Sessile glandular hairs on leaves absent. Stalked glandular hairs on leaves absent. Branched hairs on leaves absent. Sessile glandular hairs on calyx absent. Stalked glandular hairs on calyx absent. Branched hairs on calyx absent.

Ballota damascena Boiss. Plant woody. Stem hairy. Stem square. Stem prostrate. Stolons absent. Leaves petioled. Leaves simple. Leaf blade orbicular. Leaf blade $0.7 \mathrm{~cm}$ long. Leaf blade $1.3 \mathrm{~cm}$ broad. Leaf veins prominent. Leaf veins not black. Leaf margin denticulate-serrate. Leaf margin flat. Leaf apex obtuse. Leaf surface smooth. Leaves white-tomentose. Leaf venation palmate. Inflorescence dense spike-like. Flowers pedicelled. Calyx nearly regular. Calyx funnelshaped. Calyx veins prominent. Calyx teeth 5 or less. Calyx teeth not hooked. Calyx teeth not spiny-tipped. Posterior sepal 4-7 mm long. Veins in calyx tube 10 . Outer surface of petal lobes hairy. Upper lip of corolla flat-deflexed. Corolla tube included in calyx. Stamens 4. Stamens included. Staminal filaments glabrous. Filament-connective attachment not lever-like. Ovary glabrous. Style glabrous. Style gynobasic. Stigma 2 elongate arms. Stigmatic lobes equal. Fruit 4 nutlets. Eglandular hairs on leaves present. Sessile glandular hairs on leaves absent. Stalked glandular hairs on leaves present. Branched hairs on leaves absent. Sessile glandular hairs on calyx absent. Stalked glandular hairs on calyx absent. Branched hairs on calyx absent. 
Ballota kaiseri Täckh. Plant herbaceous. Stem hairy. Stem square. Stem erect. Stolons absent. Leaves petioled. Leaves simple. Leaf blade oblong-ovate. Leaf blade $1.8-2 \mathrm{~cm}$ long. Leaf blade $0.9-1.2 \mathrm{~cm}$ broad. Leaf veins prominent. Leaf veins black. Leaf margin denticulateserrate. Leaf margin flat. Leaf apex obtuse. Leaf surface rugose. Leaves not white-tomentose. Leaf venation pinnate. Inflorescence dense spikelike. Flowers sessile. Calyx nearly regular. Calyx funnel-shaped. Calyx veins prominent. Calyx teeth 8-10. Calyx teeth not hooked. Calyx teeth not spiny-tipped. Posterior sepal 6-8 $\mathrm{mm}$ long. Veins in calyx tube 10. Outer surface of petal lobes hairy. Upper lip of corolla flat-deflexed. Corolla tube much longer than calyx. Stamens 4. Stamens included. Staminal filaments hairy. Filament-connective attachment not lever-like. Ovary glabrous. Style glabrous. Style gynobasic. Stigma 2 elongate arms. Stigmatic lobes equal. Fruit 4 nutlets. Eglandular hairs on leaves present. Sessile glandular hairs on leaves present. Stalked glandular hairs on leaves present. Branched hairs on leaves absent. Sessile glandular hairs on calyx present. Stalked glandular hairs on calyx present. Branched hairs on calyx present.

Ballota pseudodictamnus (L.) Benth. Plant woody. Stem hairy. Stem square. Stem erect. Stolons absent. Leaves petioled. Leaves simple. Leaf blade oblong-ovate. Leaf blade $2.5 \mathrm{~cm}$ long. Leaf blade $2 \mathrm{~cm}$ broad. Leaf veins prominent. Leaf veins not black. Leaf margin denticulateserrate. Leaf margin flat. Leaf apex obtuse. Leaf surface rugose. Leaves not white-tomentose. Leaf venation palmate. Inflorescence dense spikelike. Flowers sessile. Calyx nearly regular. Calyx funnel-shaped. Calyx veins prominent. Calyx teeth 8-10. Calyx teeth not hooked. Calyx teeth not spiny-tipped. Posterior sepal 5-9 mm long. Veins in calyx tube 10 . Outer surface of petal lobes hairy. Upper lip of corolla flat-deflexed. Corolla tube included in calyx. Stamens 4 . Stamens included. Staminal filaments hairy. Filament-connective attachment not lever-like. Ovary glabrous. Style glabrous. Style gynobasic. Stigma 2 elongate arms. Stigmatic lobes unequal. Fruit 4 nutlets. Eglandular hairs on leaves present. Sessile glandular hairs on leaves absent. Stalked glandular hairs on leaves absent. Branched hairs on leaves absent. Sessile glandular hairs on calyx absent. Stalked glandular hairs on calyx absent. Branched hairs on calyx present.
Ballota saxatilis Siebr ex C. Presl Plant herbaceous. Stem hairy. Stem square. Stem erect. Stolons absent. Leaves petioled. Leaves simple. Leaf blade oblong-ovate. Leaf blade $1.2 \mathrm{~cm}$ long. Leaf blade $0.9 \mathrm{~cm}$ broad. Leaf veins prominent. Leaf veins black. Leaf margin denticulateserrate. Leaf margin flat. Leaf apex obtuse. Leaf surface rugose. Leaves not white-tomentose. Leaf venation pinnate. Inflorescence dense spikelike. Flowers sessile. Calyx nearly regular. Calyx funnel-shaped. Calyx veins prominent. Calyx teeth 8-10. Calyx teeth not hooked. Calyx teeth spiny-tipped. Posterior sepal 5-9 mm long. Veins in calyx tube 10 . Outer surface of petal lobes hairy. Upper lip of corolla flat-deflexed. Corolla tube included in calyx. Stamens 4. Stamens included. Staminal filaments glabrous. Filamentconnective attachment not lever-like. Ovary glabrous. Style glabrous. Style gynobasic. Stigma 2 elongate arms. Stigmatic lobes unequal. Fruit 4 nutlets. Eglandular hairs on leaves present. Sessile glandular hairs on leaves absent. Stalked glandular hairs on leaves present. Branched hairs on leaves absent. Sessile glandular hairs on calyx absent. Stalked glandular hairs on calyx present. Branched hairs on calyx absent.

Ballota undulata (Sieber ex Fresen.) Benth. Plant woody. Stem hairy. Stem square. Stem erect. Stolons absent. Leaves petioled. Leaves simple. Leaf blade cordate. Leaf blade $2.5 \mathrm{~cm}$ long. Leaf blade $2.5 \mathrm{~cm}$ broad. Leaf veins prominent. Leaf veins not black. Leaf margin denticulateserrate. Leaf margin flat. Leaf apex obtuse. Leaf surface rugose. Leaves not white-tomentose. Leaf venation palmate. Inflorescence dense spike-like. Flowers pedicelled. Calyx nearly regular. Calyx funnel-shaped. Calyx veins prominent. Calyx teeth 8-10. Calyx teeth not hooked. Calyx teeth not spiny-tipped. Posterior sepal 3-8 $\mathrm{mm}$ long. Veins in calyx tube 10 . Outer surface of petal lobes hairy. Upper lip of corolla flat-deflexed. Corolla tube included in calyx. Stamens 4. Stamens included. Staminal filaments hairy. Filament-connective attachment not lever-like. Ovary glabrous. Style glabrous. Style gynobasic. Stigma 2 elongate arms. Stigmatic lobes equal. Fruit 4 nutlets. Eglandular hairs on leaves present. Sessile glandular hairs on leaves absent. Stalked glandular hairs on leaves absent. Branched hairs on leaves present. Sessile glandular hairs on calyx absent. Stalked glandular hairs on calyx absent. Branched hairs on calyx present. 
Clerodendrum acerbianum (Vis.) Benth. Plant woody. Stem hairy. Stem square. Stem erect. Stolons absent. Leaves petioled. Leaves simple. Leaf blade cordate. Leaf blade $2.7-3.2 \mathrm{~cm}$ long. Leaf blade $0.9-2.5 \mathrm{~cm}$ broad. Leaf veins not prominent. Leaf veins not black. Leaf margin denticulate-serrate. Leaf margin flat. Leaf apex acute. Leaf surface smooth. Leaves not whitetomentose. Leaf venation pinnate. Inflorescence dense spike-like. Flowers pedicelled. Calyx nearly regular. Calyx not funnel-shaped. Calyx veins not prominent. Calyx teeth 5 or less. Calyx teeth not hooked. Calyx teeth not spiny-tipped. Posterior sepal 3-6 mm long. Veins in calyx tube 10 . Outer surface of petal lobes hairy. Upper lip of corolla nearly equal to lower lip. Corolla tube much longer than calyx. Stamens 4. Stamens exserted. Staminal filaments glabrous. Filament-connective attachment not lever-like. Ovary glabrous. Style glabrous. Style terminal. Stigma 2 elongate arms. Stigmatic lobes equal. Fruit drupe. Eglandular hairs on leaves absent. Sessile glandular hairs on leaves absent. Stalked glandular hairs on leaves absent. Branched hairs on leaves absent. Sessile glandular hairs on calyx absent. Stalked glandular hairs on calyx absent. Branched hairs on calyx absent.

Phlomoides laciniata (L.) Kamelin \& Makhm. Plant woody. Stem hairy. Stem square. Stem erect. Stolons absent. Leaves petioled. Leaves uni- to bi-pinnate. Leaf blade oblong-ovate. Leaf blade $9.5-11 \mathrm{~cm}$ long. Leaf blade $4-4.5 \mathrm{~cm}$ broad. Leaf veins not prominent. Leaf veins not black. Leaf margin denticulate-serrate. Leaf margin flat. Leaf apex obtuse. Leaf surface smooth. Leaves whitetomentose. Leaf venation pinnate. Inflorescence dense spike-like. Flowers pedicelled. Calyx nearly regular. Calyx not funnel-shaped. Calyx veins not prominent. Calyx teeth 5 or less. Calyx teeth not hooked. Calyx teeth not spiny-tipped. Posterior sepal $12-16 \mathrm{~mm}$ long. Veins in calyx tube 15 . Outer surface of petal lobes hairy. Upper lip of corolla vaulted. Corolla tube included in calyx. Stamens 4. Stamens included. Staminal filaments glabrous. Filament-connective attachment not lever-like. Ovary glabrous. Style glabrous. Style gynobasic. Stigma 2 elongate arms. Stigmatic lobes unequal. Fruit 4 nutlets. Eglandular hairs on leaves present. Sessile glandular hairs on leaves absent. Stalked glandular hairs on leaves absent. Branched hairs on leaves absent. Sessile glandular hairs on calyx absent. Stalked glandular hairs on calyx absent. Branched hairs on calyx absent.
Lallemantia royleana (Benth.) Benth. in DC. Plant herbaceous. Stem hairy. Stem square. Stem erect. Stolons absent. Leaves petioled. Leaves simple. Leaf blade oblong-ovate. Leaf blade $0.9 \mathrm{~cm}$ long. Leaf blade $0.2 \mathrm{~cm}$ broad. Leaf veins not prominent. Leaf veins not black. Leaf margin entire. Leaf margin flat. Leaf apex obtuse. Leaf surface smooth. Leaves white-tomentose. Leaf venation pinnate. Inflorescence dense spikelike. Flowers sessile. Calyx bilabiate. Calyx not funnel-shaped. Calyx veins prominent. Calyx teeth 5 or less. Calyx teeth not hooked. Calyx teeth not spiny-tipped. Posterior sepal 4-7 mm long. Veins in calyx tube 15 . Outer surface of petal lobes hairy. Upper lip of corolla flatdeflexed. Corolla tube included in calyx. Stamens 4. Stamens included. Staminal filaments hairy. Filament-connective attachment not lever-like. Ovary glabrous. Style glabrous. Style gynobasic. Stigma 2 elongate arms. Stigmatic lobes equal. Fruit 4 nutlets. Eglandular hairs on leaves present. Sessile glandular hairs on leaves absent. Stalked glandular hairs on leaves absent. Branched hairs on leaves absent. Sessile glandular hairs on calyx absent. Stalked glandular hairs on calyx absent. Branched hairs on calyx absent.

Lamium amplexicaule L. Plant herbaceous. Stem hairy. Stem square. Stem erect. Stolons absent. Leaves petioled. Leaves simple. Leaf blade cordate. Leaf blade $2-2.5 \mathrm{~cm}$ long. Leaf blade $2.2-2.5 \mathrm{~cm}$ broad. Leaf veins not prominent. Leaf veins not black. Leaf margin denticulateserrate. Leaf margin flat. Leaf apex obtuse. Leaf surface smooth. Leaves not white-tomentose. Leaf venation palmate. Inflorescence lax verticillasters. Flowers pedicelled. Calyx nearly regular. Calyx not funnel-shaped. Calyx veins prominent. Calyx teeth 5 or less. Calyx teeth not hooked. Calyx teeth not spiny-tipped. Posterior sepal 4-7 mm long. Veins in calyx tube 10. Outer surface of petal lobes hairy. Upper lip of corolla flat-deflexed. Corolla tube much longer than calyx. Stamens 4. Stamens included. Staminal filaments glabrous. Filament-connective attachment not lever-like. Ovary glabrous. Style glabrous. Style gynobasic. Stigma 2 elongate arms. Stigmatic lobes equal. Fruit 4 nutlets. Eglandular hairs on leaves absent. Sessile glandular hairs on leaves absent. Stalked glandular hairs on leaves absent. Branched hairs on leaves absent. Sessile glandular hairs on calyx absent. Stalked glandular hairs on calyx absent. Branched hairs on calyx absent. 
Lavandula atriplicifolia Benth. Plant woody. Stem hairy. Stem square. Stem erect. Stolons absent. Leaves petioled. Leaves simple. Leaf blade linear-lanceolate. Leaf blade $4.5 \mathrm{~cm}$ long. Leaf blade $2.5 \mathrm{~cm}$ broad. Leaf veins not prominent. Leaf veins not black. Leaf margin entire. Leaf margin flat. Leaf apex acute. Leaf surface smooth. Leaves white-tomentose. Leaf uninerved. Inflorescence dense spike-like. Flowers sessile. Calyx nearly regular. Calyx not funnel-shaped. Calyx veins prominent. Calyx teeth 5 or less. Calyx teeth not hooked. Calyx teeth not spiny-tipped. Posterior sepal 1-5 mm long. Veins in calyx tube 15 . Outer surface of petal lobes hairy. Upper lip of corolla flat-deflexed. Corolla tube much longer than calyx. Stamens 4. Stamens included. Staminal filaments glabrous. Filament-connective attachment not lever-like. Ovary glabrous. Style glabrous. Style gynobasic. Stigma capitate. Stigmatic lobes equal. Fruit 4 nutlets. Eglandular hairs on leaves present. Sessile glandular hairs on leaves present. Stalked glandular hairs on leaves absent. Branched hairs on leaves absent. Sessile glandular hairs on calyx present. Stalked glandular hairs on calyx absent. Branched hairs on calyx present.

Lavandula canariensis (L.) Mill. Plant woody. Stem hairy. Stem square. Stem erect. Stolons absent. Leaves sessile-subsessile. Leaves uni- to bi-pinnate. Leaf blade oblong-ovate. Leaf blade $1.8-2.8 \mathrm{~cm}$ long. Leaf blade $1-1.6 \mathrm{~cm}$ broad. Leaf veins not prominent. Leaf veins not black. Leaf margin denticulate-serrate. Leaf margin flat. Leaf apex acute. Leaf surface smooth. Leaves whitetomentose. Leaf venation pinnate. Inflorescence dense spike-like. Flowers sessile. Calyx nearly regular. Calyx not funnel-shaped. Calyx veins not prominent. Calyx teeth 5 or less. Calyx teeth not hooked. Calyx teeth not spiny-tipped. Posterior sepal 3-5 mm long. Veins in calyx tube 15. Outer surface of petal lobes glabrous. Upper lip of corolla flat-deflexed. Corolla tube much longer than calyx. Stamens 4. Stamens included. Staminal filaments glabrous. Filament-connective attachment not lever-like. Ovary glabrous. Style glabrous. Style gynobasic. Stigma capitate. Stigmatic lobes equal. Fruit 4 nutlets. Eglandular hairs on leaves present. Sessile glandular hairs on leaves absent. Stalked glandular hairs on leaves absent. Branched hairs on leaves absent. Sessile glandular hairs on calyx present. Stalked glandular hairs on calyx absent. Branched hairs on calyx absent.
Lavandula coronopifolia Poir. in Lam. Plant woody. Stem hairy. Stem square. Stem erect. Stolons absent. Leaves petioled. Leaves uni- to bi-pinnate. Leaf blade oblong-ovate. Leaf blade $2.5-2.7 \mathrm{~cm}$ long. Leaf blade $2-2.5 \mathrm{~cm}$ broad. Leaf veins not prominent. Leaf veins not black. Leaf margin denticulate-serrate. Leaf margin flat. Leaf apex acute. Leaf surface smooth. Leaves whitetomentose. Leaf venation pinnate. Inflorescence lax verticillasters. Flowers sessile. Calyx nearly regular. Calyx not funnel-shaped. Calyx veins prominent. Calyx teeth 5 or less. Calyx teeth not hooked. Calyx teeth not spiny-tipped. Posterior sepal 2-5 mm long. Veins in calyx tube 15 . Outer surface of petal lobes hairy. Upper lip of corolla flat-deflexed. Corolla tube much longer than calyx. Stamens 4. Stamens included. Staminal filaments glabrous. Filament-connective attachment not lever-like. Ovary glabrous. Style glabrous. Style gynobasic. Stigma capitate. Stigmatic lobes equal. Fruit 4 nutlets. Eglandular hairs on leaves present. Sessile glandular hairs on leaves absent. Stalked glandular hairs on leaves absent. Branched hairs on leaves absent. Sessile glandular hairs on calyx absent. Stalked glandular hairs on calyx absent. Branched hairs on calyx absent.

Lavandula pubescens Decne. Plant woody. Stem hairy. Stem square. Stem erect. Stolons absent. Leaves petioled. Leaves uni- to bipinnate. Leaf blade oblong-ovate. Leaf blade $1.7 \mathrm{~cm}$ long. Leaf blade $0.9 \mathrm{~cm}$ broad. Leaf veins not prominent. Leaf veins not black. Leaf margin denticulate-serrate. Leaf margin flat. Leaf apex acute. Leaf surface smooth. Leaves whitetomentose. Leaf venation pinnate. Inflorescence dense spike-like. Flowers sessile. Calyx bilabiate. Calyx not funnel-shaped. Calyx veins prominent. Calyx teeth 5 or less. Calyx teeth not hooked. Calyx teeth not spiny-tipped. Posterior sepal 3-6 $\mathrm{mm}$ long. Veins in calyx tube 15 . Outer surface of petal lobes glabrous. Upper lip of corolla flatdeflexed. Corolla tube much longer than calyx. Stamens 4. Stamens included. Staminal filaments glabrous. Filament-connective attachment not lever-like. Ovary glabrous. Style glabrous. Style gynobasic. Stigma capitate. Stigmatic lobes equal. Fruit 4 nutlets. Eglandular hairs on leaves absent. Sessile glandular hairs on leaves present. Stalked glandular hairs on leaves present. Branched hairs on leaves absent. Sessile glandular hairs on calyx present. Stalked glandular hairs on calyx present. Branched hairs on calyx absent. 
Leucas inflata Benth. Plant woody. Stem hairy. Stem square. Stem erect. Stolons absent. Leaves petioled. Leaves simple. Leaf blade orbicular. Leaf blade $1.2-1.6 \mathrm{~cm}$ long. Leafblade $0.8-1.1 \mathrm{~cm}$ broad. Leaf veins prominent. Leaf veins not black. Leaf margin entire. Leaf margin flat. Leaf apex acute. Leaf surface rugose. Leaves not white-tomentose. Leaf venation palmate. Inflorescence dense spikelike. Flowers sessile. Calyx bilabiate. Calyx not funnel-shaped. Calyx veins not prominent. Calyx teeth 8-10. Calyx teeth not hooked. Calyx teeth not spiny-tipped. Posterior sepal 3-6 mm long. Veins in calyx tube 10. Outer surface of petal lobes hairy. Upper lip of corolla flat-deflexed. Corolla tube much longer than calyx. Stamens 4. Stamens included. Staminal filaments glabrous. Filament-connective attachment not lever-like. Ovary glabrous. Style glabrous. Style gynobasic. Stigma 2 elongate arms. Stigmatic lobes unequal. Fruit 4 nutlets. Eglandular hairs on leaves present. Sessile glandular hairs on leaves absent. Stalked glandular hairs on leaves present. Branched hairs on leaves present. Sessile glandular hairs on calyx absent. Stalked glandular hairs on calyx present. Branched hairs on calyx present.

Leucas neuflizeana Courbon Plant herbaceous. Stem hairy. Stem square. Stem erect. Stolons absent. Leaves petioled. Leaves simple. Leaf blade linear-lanceolate. Leaf blade $1.8-2 \mathrm{~cm}$ long. Leaf blade $0.5 \mathrm{~cm}$ broad. Leaf veins prominent. Leaf veins not black. Leaf margin denticulateserrate. Leaf margin flat. Leaf apex acute. Leaf surface rugose. Leaves not white-tomentose. Leaf venation palmate. Inflorescence dense spike-like. Flowers sessile. Calyx nearly regular. Calyx not funnel-shaped. Calyx veins prominent. Calyx teeth 8-10. Calyx teeth not hooked. Calyx teeth not spiny-tipped. Posterior sepal 3-6 mm long. Veins in calyx tube 10 . Outer surface of petal lobes hairy. Upper lip of corolla flat-deflexed. Corolla tube much longer than calyx. Stamens 4 . Stamens included. Staminal filaments glabrous. Filament-connective attachment not lever-like. Ovary glabrous. Style glabrous. Style gynobasic. Stigma 2 elongate arms. Stigmatic lobes equal. Fruit 4 nutlets. Eglandular hairs on leaves present. Sessile glandular hairs on leaves absent. Stalked glandular hairs on leaves absent. Branched hairs on leaves absent. Sessile glandular hairs on calyx absent. Stalked glandular hairs on calyx absent. Branched hairs on calyx absent.

Marrubium alysson L. Plant herbaceous. Stem hairy. Stem square. Stem erect. Stolons absent. Leaves petioled. Leaves simple. Leaf blade fanshaped. Leaf blade $2.2 \mathrm{~cm}$ long. Leaf blade $2.5 \mathrm{~cm}$ broad. Leaf veins prominent. Leaf veins black. Leaf margin denticulate-serrate. Leaf margin flat. Leaf apex obtuse. Leaf surface rugose. Leaves not white-tomentose. Leaf venation palmate. Inflorescence lax verticillasters. Flowers pedicelled. Calyx nearly regular. Calyx funnelshaped. Calyx veins prominent. Calyx teeth 5 or less. Calyx teeth not hooked. Calyx teeth not spiny-tipped. Posterior sepal $6-10 \mathrm{~mm}$ long. Veins in calyx tube 10. Outer surface of petal lobes hairy. Upper lip of corolla flat-deflexed. Corolla tube included in calyx. Stamens 4. Stamens included. Staminal filaments glabrous. Filament-connective attachment not lever-like. Ovary glabrous. Style glabrous. Style gynobasic. Stigma 2 elongate arms. Stigmatic lobes unequal. Fruit 4 nutlets. Eglandular hairs on leaves present. Sessile glandular hairs on leaves absent. Stalked glandular hairs on leaves absent. Branched hairs on leaves absent. Sessile glandular hairs on calyx absent. Stalked glandular hairs on calyx absent. Branched hairs on calyx absent.

Marrubium vulgare L. Plant herbaceous. Stem hairy. Stem square. Stem erect. Stolons absent. Leaves petioled. Leaves simple. Leaf blade oblong-ovate. Leaf blade $3-3.5 \mathrm{~cm}$ long. Leaf blade $3.2-3.5 \mathrm{~cm}$ broad. Leaf veins prominent. Leaf veins black. Leaf margin denticulateserrate. Leaf margin flat. Leaf apex obtuse. Leaf surface rugose. Leaves not white-tomentose. Leaf venation palmate. Inflorescence lax verticillasters. Flowers sessile. Calyx nearly regular. Calyx not funnel-shaped. Calyx veins prominent. Calyx teeth 8-10. Calyx teeth hooked. Calyx teeth not spiny-tipped. Posterior sepal 3-6 mm long. Veins in calyx tube 10 . Outer surface of petal lobes hairy. Upper lip of corolla flat-deflexed. Corolla tube included in calyx. Stamens 4. Stamens included. Staminal filaments glabrous. Filamentconnective attachment not lever-like. Ovary glabrous. Style glabrous. Style gynobasic. Stigma 2 elongate arms. Stigmatic lobes unequal. Fruit 4 nutlets. Eglandular hairs on leaves present. Sessile glandular hairs on leaves absent. Stalked glandular hairs on leaves absent. Branched hairs on leaves present. Sessile glandular hairs on calyx absent. Stalked glandular hairs on calyx absent. Branched hairs on calyx present.

Mentha longifolia (L.) Huds. var. schimperi 
(Briq.) Briq. Plant herbaceous. Stem glabrous. Stem square. Stem erect. Stolons present. Leaves sessile-subsessile. Leaves simple. Leaf blade oblong-ovate. Leaf blade $5.5-6.5 \mathrm{~cm}$ long. Leaf blade $2-2.6 \mathrm{~cm}$ broad. Leaf veins prominent. Leaf veins not black. Leaf margin denticulate-serrate. Leaf margin flat. Leaf apex acute. Leaf surface smooth. Leaves not white-tomentose. Leaf venation pinnate. Inflorescence dense spike-like. Flowers sessile. Calyx nearly regular. Calyx not funnel-shaped. Calyx veins not prominent. Calyx teeth 5 or less. Calyx teeth not hooked. Calyx teeth not spiny-tipped. Posterior sepal 1-4 mm long. Veins in calyx tube 10 . Outer surface of petal lobes glabrous. Upper lip of corolla nearly equal to lower lip. Corolla tube much longer than calyx. Stamens 4. Stamens exserted. Staminal filaments glabrous. Filament-connective attachment not lever-like. Ovary glabrous. Style glabrous. Style gynobasic. Stigma 2 elongate arms. Stigmatic lobes equal. Fruit 4 nutlets. Eglandular hairs on leaves present. Sessile glandular hairs on leaves absent. Stalked glandular hairs on leaves absent. Branched hairs on leaves absent. Sessile glandular hairs on calyx present. Stalked glandular hairs on calyx absent. Branched hairs on calyx absent.

Mentha longifolia (L.) Huds. ssp. typhoides (Briq.) Harley Plant herbaceous. Stem glabrous. Stem square. Stem erect. Stolons present. Leaves petioled. Leaves simple. Leaf blade oblongovate. Leaf blade $3.6-4.2 \mathrm{~cm}$ long. Leaf blade $1.7-2 \mathrm{~cm}$ broad. Leaf veins prominent. Leaf veins not black. Leaf margin denticulate-serrate. Leaf margin flat. Leaf apex acute. Leaf surface smooth. Leaves not white-tomentose. Leaf venation pinnate. Inflorescence dense spike-like. Flowers sessile. Calyx nearly regular. Calyx not funnelshaped. Calyx veins not prominent. Calyx teeth 5 or less. Calyx teeth not hooked. Calyx teeth not spiny-tipped. Posterior sepal 1-4 mm long. Veins in calyx tube 10 . Outer surface of petal lobes glabrous. Upper lip of corolla nearly equal to lower lip. Corolla tube much longer than calyx. Stamens 4. Stamens exserted. Staminal filaments glabrous. Filament-connective attachment not lever-like. Ovary glabrous. Style glabrous. Style gynobasic. Stigma 2 elongate arms. Stigmatic lobes equal. Fruit 4 nutlets. Eglandular hairs on leaves present. Sessile glandular hairs on leaves absent. Stalked glandular hairs on leaves present. Branched hairs on leaves absent. Sessile glandular hairs on calyx present. Stalked glandular hairs on calyx absent. Branched hairs on calyx absent.
Mentha pulegium L. Plant herbaceous. Stem glabrous. Stem square. Stem prostrate. Stolons present. Leaves petioled. Leaves simple. Leaf blade oblong-ovate. Leaf blade $1.5-2.5 \mathrm{~cm}$ long. Leaf blade $0.7-1.2 \mathrm{~cm}$ broad. Leaf veins not prominent. Leaf veins not black. Leaf margin denticulate-serrate. Leaf margin flat. Leaf apex obtuse. Leaf surface smooth. Leaves not whitetomentose. Leaf venation pinnate. Inflorescence lax verticillasters. Flowers pedicelled. Calyx nearly regular. Calyx funnel-shaped. Calyx veins not prominent. Calyx teeth 5 or less. Calyx teeth not hooked. Calyx teeth not spiny-tipped. Posterior sepal 2-3 mm long. Veins in calyx tube 10. Outer surface of petal lobes glabrous. Upper lip of corolla nearly equal to lower lip. Corolla tube included in calyx. Stamens 4. Stamens exserted. Staminal filaments hairy. Filament-connective attachment not lever-like. Ovary glabrous. Style hairy. Style gynobasic. Stigma 2 elongate arms. Stigmatic lobes equal. Fruit 4 nutlets. Eglandular hairs on leaves absent. Sessile glandular hairs on leaves absent. Stalked glandular hairs on leaves absent. Branched hairs on leaves absent. Sessile glandular hairs on calyx present. Stalked glandular hairs on calyx absent. Branched hairs on calyx absent.

Micromeria imbricata (Forssk.) C. Chr. Plant woody. Stem hairy. Stem square. Stem erect. Stolons absent. Leaves petioled. Leaves simple. Leaf blade oblong-ovate. Leaf blade $0.7-0.9 \mathrm{~cm}$ long. Leaf blade $0.2-0.4 \mathrm{~cm}$ broad. Leaf veins prominent. Leaf veins not black. Leaf margin entire. Leaf margin revolute. Leaf apex acute. Leaf surface rugose. Leaves not white-tomentose. Leaf venation pinnate. Inflorescence lax verticillasters. Flowers pedicelled. Calyx nearly regular. Calyx not funnel-shaped. Calyx veins prominent. Calyx teeth 5 or less. Calyx teeth not hooked. Calyx teeth not spiny-tipped. Posterior sepal 2-4 mm long. Veins in calyx tube 13 . Outer surface of petal lobes glabrous. Upper lip of corolla flat-deflexed. Corolla tube much longer than calyx. Stamens 4 . Stamens included. Staminal filaments glabrous. Filament-connective attachment not lever-like. Ovary glabrous. Style glabrous. Style gynobasic. Stigma 2 elongate arms. Stigmatic lobes equal. Fruit 4 nutlets. Eglandular hairs on leaves present. Sessile glandular hairs on leaves absent. Stalked glandular hairs on leaves absent. Branched hairs on leaves absent. Sessile glandular hairs on calyx present. Stalked glandular hairs on calyx absent. Branched hairs on calyx absent. 
Micromeria myrtifolia Boiss. \& Hohen. in Boiss. Plant woody. Stem hairy. Stem square. Stem erect. Stolons absent. Leaves petioled. Leaves simple. Leaf blade oblong-ovate. Leaf blade $0.6 \mathrm{~cm}$ long. Leaf blade $0.1 \mathrm{~cm}$ broad. Leaf veins prominent. Leaf veins not black. Leaf margin entire. Leaf margin revolute. Leaf apex acute. Leaf surface rugose. Leaves not white-tomentose. Leaf venation pinnate. Inflorescence dense spikelike. Flowers pedicelled. Calyx nearly regular. Calyx not funnel-shaped. Calyx veins prominent. Calyx teeth 5 or less. Calyx teeth not hooked. Calyx teeth not spiny-tipped. Posterior sepal 2-4 $\mathrm{mm}$ long. Veins in calyx tube 13 . Outer surface of petal lobes glabrous. Upper lip of corolla flatdeflexed. Corolla tube much longer than calyx. Stamens 4. Stamens included. Staminal filaments glabrous. Filament-connective attachment not lever-like. Ovary glabrous. Style glabrous. Style gynobasic. Stigma 2 elongate arms. Stigmatic lobes equal. Fruit 4 nutlets. Eglandular hairs on leaves present. Sessile glandular hairs on leaves absent. Stalked glandular hairs on leaves absent. Branched hairs on leaves absent. Sessile glandular hairs on calyx present. Stalked glandular hairs on calyx absent. Branched hairs on calyx absent.

Micromeria nervosa (Desf.) Benth. Plant woody. Stem hairy. Stem square. Stem erect. Stolons absent. Leaves petioled. Leaves simple. Leaf blade oblong-ovate. Leaf blade $1.1 \mathrm{~cm}$ long. Leaf blade $0.6 \mathrm{~cm}$ broad. Leaf veins prominent. Leaf veins not black. Leaf margin entire. Leaf margin revolute. Leaf apex acute. Leaf surface rugose. Leaves not white-tomentose. Leaf venation pinnate. Inflorescence lax verticillasters. Flowers pedicelled. Calyx nearly regular. Calyx not funnel-shaped. Calyx veins prominent. Calyx teeth 5 or less. Calyx teeth not hooked. Calyx teeth not spiny-tipped. Posterior sepal 2-4 mm long. Veins in calyx tube 13 . Outer surface of petal lobes glabrous. Upper lip of corolla flat-deflexed. Corolla tube much longer than calyx. Stamens 4. Stamens included. Staminal filaments glabrous. Filament-connective attachment not lever-like. Ovary glabrous. Style glabrous. Style gynobasic. Stigma 2 elongate arms. Stigmatic lobes unequal. Fruit 4 nutlets. Eglandular hairs on leaves present. Sessile glandular hairs on leaves absent. Stalked glandular hairs on leaves absent. Branched hairs on leaves absent. Sessile glandular hairs on calyx present. Stalked glandular hairs on calyx absent. Branched hairs on calyx absent.
Micromeria sinaica Benth. Plant woody. Stem hairy. Stem square. Stem erect. Stolons absent. Leaves sessile-subsessile. Leaves simple. Leaf blade oblong-ovate. Leaf blade $0.7-0.8 \mathrm{~cm}$ long. Leaf blade $0.4-0.5 \mathrm{~cm}$ broad. Leaf veins not prominent. Leaf veins not black. Leaf margin entire. Leaf margin revolute. Leaf apex acute. Leaf surface rugose. Leaves not white-tomentose. Leaf venation pinnate. Inflorescence lax verticillasters. Flowers sessile. Calyx nearly regular. Calyx not funnel-shaped. Calyx veins prominent. Calyx teeth 5 or less. Calyx teeth not hooked. Calyx teeth not spiny-tipped. Posterior sepal 1-3 mm long. Veins in calyx tube 13. Outer surface of petal lobes glabrous. Upper lip of corolla flat-deflexed. Corolla tube much longer than calyx. Stamens 4 . Stamens included. Staminal filaments glabrous. Filament-connective attachment not lever-like. Ovary glabrous. Style glabrous. Style gynobasic. Stigma 2 elongate arms. Stigmatic lobes equal. Fruit 4 nutlets. Eglandular hairs on leaves present. Sessile glandular hairs on leaves present. Stalked glandular hairs on leaves absent. Branched hairs on leaves absent. Sessile glandular hairs on calyx present. Stalked glandular hairs on calyx absent. Branched hairs on calyx absent.

Nepeta septemcrenata Ehrenb. ex Benth. Plant woody. Stem hairy. Stem square. Stem erect. Stolons absent. Leaves petioled. Leaves simple. Leaf blade oblong-ovate. Leaf blade $1.7 \mathrm{~cm}$ long. Leaf blade $1.6 \mathrm{~cm}$ broad. Leaf veins not prominent. Leaf veins not black. Leaf margin denticulateserrate. Leaf margin flat. Leaf apex obtuse. Leaf surface smooth. Leaves white-tomentose. Leaf venation palmate. Inflorescence lax verticillasters. Flowers pedicelled. Calyx bilabiate. Calyx not funnel-shaped. Calyx veins prominent. Calyx teeth 5 or less. Calyx teeth not hooked. Calyx teeth not spiny-tipped. Posterior sepal 4-6 mm long. Veins in calyx tube 15 . Outer surface of petal lobes glabrous. Upper lip of corolla flat-deflexed. Corolla tube much longer than calyx. Stamens 4 . Stamens exserted. Staminal filaments glabrous. Filament-connective attachment not lever-like. Ovary glabrous. Style glabrous. Style gynobasic. Stigma 2 elongate arms. Stigmatic lobes equal. Fruit 4 nutlets. Eglandular hairs on leaves present. Sessile glandular hairs on leaves present. Stalked glandular hairs on leaves absent. Branched hairs on leaves absent. Sessile glandular hairs on calyx present. Stalked glandular hairs on calyx absent. Branched hairs on calyx absent. 
Ocimum forsskaolii Benth. Plant woody. Stem hairy. Stem square. Stem erect. Stolons absent. Leaves petioled. Leaves simple. Leaf blade oblong-ovate. Leaf blade $2.5-2.8 \mathrm{~cm}$ long. Leaf blade $1.8-2 \mathrm{~cm}$ broad. Leaf veins not prominent. Leaf veins not black. Leaf margin entire. Leaf margin flat. Leaf apex acute. Leaf surface smooth. Leaves white-tomentose. Leaf venation pinnate. Inflorescence lax verticillasters. Flowers pedicelled. Calyx bilabiate. Calyx not funnel-shaped. Calyx veins prominent. Calyx teeth 5 or less. Calyx teeth not hooked. Calyx teeth not spiny-tipped. Posterior sepal 2-5 mm long. Veins in calyx tube 10 . Outer surface of petal lobes hairy. Upper lip of corolla flatdeflexed. Corolla tube included in calyx. Stamens 4. Stamens exserted. Staminal filaments glabrous. Filament-connective attachment not lever-like. Ovary glabrous. Style glabrous. Style gynobasic. Stigma 2 elongate arms. Stigmatic lobes equal. Fruit 4 nutlets. Eglandular hairs on leaves present. Sessile glandular hairs on leaves absent. Stalked glandular hairs on leaves absent. Branched hairs on leaves absent. Sessile glandular hairs on calyx absent. Stalked glandular hairs on calyx absent. Branched hairs on calyx absent.

Origanum syriacum L. ssp. sinaicum (Boiss.) Greuter \& Burdet Plant herbaceous. Stem glabrous. Stem square. Stem erect. Stolons absent. Leaves sessile-subsessile. Leaves simple. Leaf blade oblong-ovate. Leaf blade $1.4-1.6 \mathrm{~cm}$ long. Leaf blade $0.8-1 \mathrm{~cm}$ broad. Leaf veins prominent. Leaf veins not black. Leaf margin entire. Leaf margin flat. Leaf apex obtuse. Leaf surface smooth. Leaves white-tomentose. Leaf venation pinnate. Inflorescence terminal globose aggregate. Flowers sessile. Calyx nearly regular. Calyx not funnel-shaped. Calyx veins not prominent. Calyx teeth 5 or less. Calyx teeth not hooked. Calyx teeth not spiny-tipped. Posterior sepal 1-3 mm long. Veins in calyx tube 5. Outer surface of petal lobes hairy. Upper lip of corolla flat-deflexed. Corolla tube included in calyx. Stamens 4. Stamens included. Staminal filaments glabrous. Filament-connective attachment not lever-like. Ovary glabrous. Style glabrous. Style gynobasic. Stigma capitate. Stigmatic lobes equal. Fruit 4 nutlets. Eglandular hairs on leaves present. Sessile glandular hairs on leaves absent. Stalked glandular hairs on leaves present. Branched hairs on leaves absent. Sessile glandular hairs on calyx present. Stalked glandular hairs on calyx absent. Branched hairs on calyx present.
Orthosiphon pallidus Royle ex Benth. in Hook. Plant herbaceous. Stem hairy. Stem square. Stem erect. Stolons absent. Leaves petioled. Leaves simple. Leaf blade oblong-ovate. Leaf blade $0.8-1.8 \mathrm{~cm}$ long. Leaf blade $0.5-1.5 \mathrm{~cm}$ broad. Leaf veins not prominent. Leaf veins not black. Leaf margin denticulate-serrate. Leaf margin flat. Leaf apex obtuse. Leaf surface smooth. Leaves not white-tomentose. Leaf venation pinnate. Inflorescence lax verticillasters. Flowers pedicelled. Calyx bilabiate. Calyx not funnel-shaped. Calyx veins prominent. Calyx teeth 5 or less. Calyx teeth not hooked. Calyx teeth not spiny-tipped. Posterior sepal 2-2.5 $\mathrm{mm}$ long. Veins in calyx tube 10 . Outer surface of petal lobes glabrous. Upper lip of corolla flatdeflexed. Corolla tube included in calyx. Stamens 4. Stamens exserted. Staminal filaments glabrous. Filament-connective attachment not lever-like. Ovary glabrous. Style glabrous. Style gynobasic. Stigma capitate. Stigmatic lobes equal. Fruit 4 nutlets. Eglandular hairs on leaves absent. Sessile glandular hairs on leaves absent. Stalked glandular hairs on leaves absent. Branched hairs on leaves absent. Sessile glandular hairs on calyx present. Stalked glandular hairs on calyx present. Branched hairs on calyx absent.

Otostigia fruticosa (Forssk.) Scheinf. ex Penzig ssp. fruticosa Plant woody. Stem hairy. Stem square. Stem erect. Stolons absent. Leaves petioled. Leaves simple. Leaf blade oblong-ovate. Leaf blade $2.6 \mathrm{~cm}$ long. Leaf blade $2 \mathrm{~cm}$ broad. Leaf veins prominent. Leaf veins not black. Leaf margin denticulate-serrate. Leaf margin flat. Leaf apex obtuse. Leaf surface smooth. Leaves whitetomentose. Leaf venation palmate. Inflorescence lax verticillasters. Flowers pedicelled. Calyx bilabiate. Calyx funnel-shaped. Calyx veins prominent. Calyx teeth 5 or less. Calyx teeth not hooked. Calyx teeth not spiny-tipped. Posterior sepal 6-9 mm long. Veins in calyx tube 10. Outer surface of petal lobes glabrous. Upper lip of corolla vaulted. Corolla tube included in calyx. Stamens 4. Stamens included. Staminal filaments glabrous. Filament-connective attachment not lever-like. Ovary glabrous. Style glabrous. Style gynobasic. Stigma 2 elongate arms. Stigmatic lobes equal. Fruit 4 nutlets. Eglandular hairs on leaves present. Sessile glandular hairs on leaves absent. Stalked glandular hairs on leaves present. Branched hairs on leaves absent. Sessile glandular hairs on calyx absent. Stalked glandular hairs on calyx absent. Branched hairs on calyx absent. 
Otostegia fruticosa (Forssk.) Penzig ssp. schimperi (Benth.) Sebald Plant woody. Stem hairy. Stem square. Stem erect. Stolons absent. Leaves petioled. Leaves simple. Leaf blade oblong-ovate. Leaf blade $3-3.5 \mathrm{~cm}$ long. Leaf blade $2-2.3 \mathrm{~cm}$ broad. Leaf veins prominent. Leaf veins not black. Leaf margin sinuate. Leaf margin flat. Leaf apex obtuse. Leaf surface smooth. Leaves white-tomentose. Leaf venation palmate. Inflorescence lax verticillasters. Flowers pedicelled. Calyx bilabiate. Calyx funnel-shaped. Calyx veins prominent. Calyx teeth 5 or less. Calyx teeth not hooked. Calyx teeth not spiny-tipped. Posterior sepal 4-10 mm long. Veins in calyx tube 10. Outer surface of petal lobes glabrous. Upper lip of corolla vaulted. Corolla tube included in calyx. Stamens 4. Stamens included. Staminal filaments glabrous. Filament-connective attachment not lever-like. Ovary glabrous. Style glabrous. Style gynobasic. Stigma 2 elongate arms. Stigmatic lobes equal. Fruit 4 nutlets. Eglandular hairs on leaves present. Sessile glandular hairs on leaves absent. Stalked glandular hairs on leaves absent. Branched hairs on leaves absent. Sessile glandular hairs on calyx absent. Stalked glandular hairs on calyx absent. Branched hairs on calyx absent.

Phlomis aurea Decne. Plant woody. Stem hairy. Stem square. Stem erect. Stolons absent. Leaves petioled. Leaves simple. Leaf blade oblong-ovate. Leaf blade $6.7 \mathrm{~cm}$ long. Leaf blade $2.6 \mathrm{~cm}$ broad. Leaf veins prominent. Leaf veins not black. Leaf margin entire. Leaf margin flat. Leaf apex obtuse. Leaf surface rugose. Leaves not whitetomentose. Leaf venation pinnate. Inflorescence lax verticillasters. Flowers pedicelled. Calyx nearly regular. Calyx funnel-shaped. Calyx veins prominent. Calyx teeth 8-10. Calyx teeth not hooked. Calyx teeth not spiny-tipped. Posterior sepal 9-16 mm long. Veins in calyx tube 10. Outer surface of petal lobes hairy. Upper lip of corolla vaulted. Corolla tube included in calyx. Stamens 4. Stamens included. Staminal filaments glabrous. Filament-connective attachment not lever-like. Ovary glabrous. Style glabrous. Style gynobasic. Stigma 2 elongate arms. Stigmatic lobes unequal. Fruit 4 nutlets. Eglandular hairs on leaves absent. Sessile glandular hairs on leaves absent. Stalked glandular hairs on leaves absent. Branched hairs on leaves present. Sessile glandular hairs on calyx absent. Stalked glandular hairs on calyx absent. Branched hairs on calyx present.

Phlomis floccosa D. Don Plant woody. Stem hairy. Stem square. Stem erect. Stolons absent. Leaves petioled. Leaves simple. Leaf blade oblong-ovate. Leaf blade $7-9 \mathrm{~cm}$ long. Leaf blade $2.5-3 \mathrm{~cm}$ broad. Leaf veins prominent. Leaf veins not black. Leaf margin denticulateserrate. Leaf margin flat. Leaf apex obtuse. Leaf surface rugose. Leaves not white-tomentose. Leaf venation palmate. Inflorescence lax verticillasters. Flowers pedicelled. Calyx nearly regular. Calyx funnel-shaped. Calyx veins prominent. Calyx teeth 8-10. Calyx teeth hooked. Calyx teeth not spiny-tipped. Posterior sepal 14-18 mm long. Veins in calyx tube 10 . Outer surface of petal lobes hairy. Upper lip of corolla vaulted. Corolla tube included in calyx. Stamens 4. Stamens included. Staminal filaments glabrous. Filamentconnective attachment not lever-like. Ovary glabrous. Style hairy. Style gynobasic. Stigma 2 elongate arms. Stigmatic lobes unequal. Fruit 4 nutlets. Eglandular hairs on leaves present. Sessile glandular hairs on leaves absent. Stalked glandular hairs on leaves absent. Branched hairs on leaves present. Sessile glandular hairs on calyx absent. Stalked glandular hairs on calyx absent. Branched hairs on calyx present.

Prasium majus L. Plant herbaceous. Stem glabrous. Stem square. Stem erect. Stolons absent. Leaves petioled. Leaves simple. Leaf blade oblong-ovate. Leaf blade $2.5-3 \mathrm{~cm}$ long. Leaf blade $1.7-1.9 \mathrm{~cm}$ broad. Leaf veins prominent. Leaf veins not black. Leaf margin denticulateserrate. Leaf margin flat. Leaf apex acute. Leaf surface smooth. Leaves not white-tomentose. Leaf venation pinnate. Inflorescence dense spikelike. Flowers pedicelled. Calyx bilabiate. Calyx funnel-shaped. Calyx veins not prominent. Calyx teeth 5 or less. Calyx teeth not hooked. Calyx teeth not spiny-tipped. Posterior sepal 10-12 $\mathrm{mm}$ long. Veins in calyx tube 13 . Outer surface of petal lobes glabrous. Upper lip of corolla flatdeflexed. Corolla tube included in calyx. Stamens 4. Stamens included. Staminal filaments glabrous. Filament-connective attachment not lever-like. Ovary glabrous. Style hairy. Style gynobasic. Stigma 2 elongate arms. Stigmatic lobes unequal. Fruit 4 nutlets. Eglandular hairs on leaves absent. Sessile glandular hairs on leaves absent. Stalked glandular hairs on leaves absent. Branched hairs on leaves absent. Sessile glandular hairs on calyx absent. Stalked glandular hairs on calyx absent. Branched hairs on calyx absent.

Salvia aegyptiaca L. Plant woody. Stem 
hairy. Stem square. Stem erect. Stolons absent. Leaves petioled. Leaves simple. Leaf blade linear-lanceolate. Leaf blade $2.5-3.2 \mathrm{~cm}$ long. Leaf blade $0.5 \mathrm{~cm}$ broad. Leaf veins prominent. Leaf veins not black. Leaf margin sinuate. Leaf margin revolute. Leaf apex acute. Leaf surface rugose. Leaves not white-tomentose. Leaf venation pinnate. Inflorescence lax verticillasters. Flowers pedicelled. Calyx bilabiate. Calyx not funnel-shaped. Calyx veins prominent. Calyx teeth 5 or less. Calyx teeth not hooked. Calyx teeth not spiny-tipped. Posterior sepal 3-5 mm long. Veins in calyx tube 15. Outer surface of petal lobes hairy. Upper lip of corolla flatdeflexed. Corolla tube included in calyx. Stamens 2. Stamens exserted. Staminal filaments glabrous. Filament-connective attachment lever-like. Ovary hairy. Style glabrous. Style gynobasic. Stigma 2 elongate arms. Stigmatic lobes unequal. Fruit 4 nutlets. Eglandular hairs on leaves present. Sessile glandular hairs on leaves absent. Stalked glandular hairs on leaves absent. Branched hairs on leaves absent. Sessile glandular hairs on calyx absent. Stalked glandular hairs on calyx present. Branched hairs on calyx absent.

Salvia deserti Decne. Plant woody. Stem hairy. Stem square. Stem erect. Stolons absent. Leaves petioled. Leaves simple. Leaf blade linearlanceolate. Leaf blade 1.6-1.9cm long. Leaf blade $0.4 \mathrm{~cm}$ broad. Leaf veins not prominent. Leaf veins not black. Leaf margin sinuate. Leaf margin revolute. Leaf apex obtuse. Leaf surface rugose. Leaves not white-tomentose. Leaf venation pinnate. Inflorescence lax verticillasters. Flowers pedicelled. Calyx bilabiate. Calyx not funnelshaped. Calyx veins not prominent. Calyx teeth 5 or less. Calyx teeth not hooked. Calyx teeth not spiny-tipped. Posterior sepal 2-4 mm long. Veins in calyx tube 10 . Outer surface of petal lobes hairy. Upper lip of corolla flat-deflexed. Corolla tube included in calyx. Stamens 2. Stamens exserted. Staminal filaments glabrous. Filament-connective attachment lever-like. Ovary glabrous. Style glabrous. Style gynobasic. Stigma 2 elongate arms. Stigmatic lobes unequal. Fruit 4 nutlets. Eglandular hairs on leaves present. Sessile glandular hairs on leaves absent. Stalked glandular hairs on leaves present. Branched hairs on leaves absent. Sessile glandular hairs on calyx absent. Stalked glandular hairs on calyx absent. Branched hairs on calyx present.

Salvia dominica L. Plant woody. Stem hairy.
Stem square. Stem erect. Stolons absent. Leaves petioled. Leaves simple. Leaf blade oblong-ovate. Leaf blade $3-6.3 \mathrm{~cm}$ long. Leaf blade $1.5-2.9 \mathrm{~cm}$ broad. Leaf veins prominent. Leaf veins not black. Leaf margin denticulate-serrate. Leaf margin flat. Leaf apex acute. Leaf surface rugose. Leaves white-tomentose. Leaf venation pinnate. Inflorescence lax verticillasters. Flowers sessile. Calyx bilabiate. Calyx funnel-shaped. Calyx veins prominent. Calyx teeth 5 or less. Calyx teeth not hooked. Calyx teeth not spiny-tipped. Posterior sepal 7-10 mm long. Veins in calyx tube 10. Outer surface of petal lobes hairy. Upper lip of corolla vaulted. Corolla tube included in calyx. Stamens 2. Stamens included. Staminal filaments hairy. Filament-connective attachment lever-like. Ovary glabrous. Style hairy. Style gynobasic. Stigma 2 elongate arms. Stigmatic lobes unequal. Fruit 4 nutlets. Eglandular hairs on leaves present. Sessile glandular hairs on leaves present. Stalked glandular hairs on leaves present. Branched hairs on leaves absent. Sessile glandular hairs on calyx present. Stalked glandular hairs on calyx present. Branched hairs on calyx absent.

Salvia lanigera Poir. in Lam. Plant woody. Stem hairy. Stem square. Stem erect. Stolons absent. Leaves petioled. Leaves simple. Leaf blade oblong-ovate. Leaf blade $3.5 \mathrm{~cm}$ long. Leaf blade $1.5 \mathrm{~cm}$ broad. Leaf veins not prominent. Leaf veins not black. Leaf margin denticulateserrate. Leaf margin revolute. Leaf apex obtuse. Leaf surface rugose. Leaves not white-tomentose. Leaf venation pinnate. Inflorescence lax verticillasters. Flowers sessile. Calyx bilabiate. Calyx funnel-shaped. Calyx veins not prominent. Calyx teeth 5 or less. Calyx teeth not hooked. Calyx teeth not spiny-tipped. Posterior sepal 3-5 $\mathrm{mm}$ long. Veins in calyx tube 13 . Outer surface of petal lobes hairy. Upper lip of corolla vaulted. Corolla tube much longer than calyx. Stamens 2. Stamens included. Staminal filaments glabrous. Filament-connective attachment lever-like. Ovary glabrous. Style glabrous. Style gynobasic. Stigma 2 elongate arms. Stigmatic lobes unequal. Fruit 4 nutlets. Eglandular hairs on leaves present. Sessile glandular hairs on leaves absent. Stalked glandular hairs on leaves absent. Branched hairs on leaves absent. Sessile glandular hairs on calyx present. Stalked glandular hairs on calyx absent. Branched hairs on calyx absent.

Salvia multicaulis Vahl Plant woody. Stem hairy. Stem square. Stem erect. Stolons absent. 
Leaves petioled. Leaves simple. Leaf blade oblong-ovate. Leaf blade $11 \mathrm{~cm}$ long. Leaf blade $7 \mathrm{~cm}$ broad. Leaf veins not prominent. Leaf veins not black. Leaf margin entire. Leaf margin flat. Leaf apex obtuse. Leaf surface smooth. Leaves white-tomentose. Leaf venation pinnate. Inflorescence lax verticillasters. Flowers pedicelled. Calyx bilabiate. Calyx not funnelshaped. Calyx veins not prominent. Calyx teeth 5 or less. Calyx teeth not hooked. Calyx teeth not spiny-tipped. Posterior sepal 7-10 mm long. Veins in calyx tube 13 . Outer surface of petal lobes glabrous. Upper lip of corolla vaulted. Corolla tube much longer than calyx. Stamens 2 . Stamens included. Staminal filaments glabrous. Filament-connective attachment lever-like. Ovary glabrous. Style glabrous. Style gynobasic. Stigma 2 elongate arms. Stigmatic lobes unequal. Fruit 4 nutlets. Eglandular hairs on leaves present. Sessile glandular hairs on leaves absent. Stalked glandular hairs on leaves absent. Branched hairs on leaves absent. Sessile glandular hairs on calyx present. Stalked glandular hairs on calyx present. Branched hairs on calyx absent.

Salvia palaestina Benth. Plant woody. Stem hairy. Stem square. Stem erect. Stolons absent. Leaves petioled. Leaves partially pinnate. Leaf blade oblong-ovate. Leaf blade $9.5 \mathrm{~cm}$ long. Leaf blade $5 \mathrm{~cm}$ broad. Leaf veins not prominent. Leaf veins not black. Leaf margin sinuate. Leaf margin flat. Leaf apex acute. Leaf surface rugose. Leaves not white-tomentose. Leaf venation pinnate. Inflorescence lax verticillasters. Flowers pedicelled. Calyx bilabiate. Calyx not funnelshaped. Calyx veins prominent. Calyx teeth 5 or less. Calyx teeth not hooked. Calyx teeth spiny-tipped. Posterior sepal 15-20 mm long. Veins in calyx tube 13 . Outer surface of petal lobes glabrous. Upper lip of corolla vaulted. Corolla tube much longer than calyx. Stamens 2. Stamens included. Staminal filaments glabrous. Filament-connective attachment lever-like. Ovary glabrous. Style glabrous. Style gynobasic. Stigma 2 elongate arms. Stigmatic lobes unequal. Fruit 4 nutlets. Eglandular hairs on leaves present. Sessile glandular hairs on leaves present. Stalked glandular hairs on leaves absent. Branched hairs on leaves absent. Sessile glandular hairs on calyx present. Stalked glandular hairs on calyx absent. Branched hairs on calyx absent.

Salvia sclarea L. Plant herbaceous. Stem hairy. Stem square. Stem erect. Stolons absent. Leaves petioled. Leaves simple. Leaf blade oblong-ovate. Leaf blade $6-10.5 \mathrm{~cm}$ long. Leaf blade $6-11 \mathrm{~cm}$ broad. Leaf veins prominent. Leaf veins not black. Leaf margin sinuate. Leaf margin flat. Leaf apex acute. Leaf surface rugose. Leaves not whitetomentose. Leaf venation pinnate. Inflorescence dense spike-like. Flowers pedicelled. Calyx bilabiate. Calyx not funnel-shaped. Calyx veins prominent. Calyx teeth 5 or less. Calyx teeth not hooked. Calyx teeth spiny-tipped. Posterior sepal $10 \mathrm{~mm}$ long. Veins in calyx tube 10 . Outer surface of petal lobes hairy. Upper lip of corolla vaulted. Corolla tube included in calyx. Stamens 2. Stamens included. Staminal filaments glabrous. Filament-connective attachment lever-like. Ovary glabrous. Style glabrous. Style gynobasic. Stigma 2 elongate arms. Stigmatic lobes unequal. Fruit 4 nutlets. Eglandular hairs on leaves present. Sessile glandular hairs on leaves present. Stalked glandular hairs on leaves absent. Branched hairs on leaves absent. Sessile glandular hairs on calyx absent. Stalked glandular hairs on calyx absent. Branched hairs on calyx present.

Salvia spinosa L. Plant woody. Stem hairy. Stem square. Stem erect. Stolons absent. Leaves petioled. Leaves simple. Leaf blade cordate. Leaf blade $14.5 \mathrm{~cm}$ long. Leaf blade $9.5 \mathrm{~cm}$ broad. Leaf veins not prominent. Leaf veins not black. Leaf margin denticulate-serrate. Leaf margin flat. Leaf apex acute. Leaf surface rugose. Leaves not whitetomentose. Leaf venation pinnate. Inflorescence lax verticillasters. Flowers pedicelled. Calyx bilabiate. Calyx not funnel-shaped. Calyx veins prominent. Calyx teeth 5 or less. Calyx teeth not hooked. Calyx teeth spiny-tipped. Posterior sepal $15-20 \mathrm{~mm}$ long. Veins in calyx tube 10. Outer surface of petal lobes hairy. Upper lip of corolla vaulted. Corolla tube included in calyx. Stamens 2. Stamens exserted. Staminal filaments glabrous. Filament-connective attachment lever-like. Ovary glabrous. Style glabrous. Style gynobasic. Stigma 2 elongate arms. Stigmatic lobes unequal. Fruit 4 nutlets. Eglandular hairs on leaves present. Sessile glandular hairs on leaves absent. Stalked glandular hairs on leaves present. Branched hairs on leaves present. Sessile glandular hairs on calyx absent. Stalked glandular hairs on calyx absent. Branched hairs on calyx present.

Salvia verbenaca L. Plant woody. Stem hairy. Stem square. Stem erect. Stolons absent. Leaves petioled. Leaves partially pinnate. Leaf blade 
oblong-ovate. Leaf blade $7.5-11.5 \mathrm{~cm}$ long. Leaf blade $5.8-7.5 \mathrm{~cm}$ broad. Leaf veins prominent. Leaf veins not black. Leaf margin denticulateserrate. Leaf margin flat. Leaf apex acute. Leaf surface rugose. Leaves not white-tomentose. Leaf venation pinnate. Inflorescence lax verticillasters. Flowers sessile. Calyx bilabiate. Calyx not funnel-shaped. Calyx veins not prominent. Calyx teeth 5 or less. Calyx teeth not hooked. Calyx teeth not spiny-tipped. Posterior sepal 5-8 mm long. Veins in calyx tube 13 . Outer surface of petal lobes glabrous. Upper lip of corolla vaulted. Corolla tube included in calyx. Stamens 2. Stamens included. Staminal filaments glabrous. Filament-connective attachment not lever-like. Ovary glabrous. Style glabrous. Style gynobasic. Stigma 2 elongate arms. Stigmatic lobes unequal. Fruit 4 nutlets. Eglandular hairs on leaves present. Sessile glandular hairs on leaves absent. Stalked glandular hairs on leaves absent. Branched hairs on leaves absent. Sessile glandular hairs on calyx absent. Stalked glandular hairs on calyx absent. Branched hairs on calyx absent.

Stachys aegyptiaca Pers. Plant woody. Stem hairy. Stem square. Stem erect. Stolons absent. Leaves sessile-subsessile. Leaves simple. Leaf blade linear-lanceolate. Leaf blade $2.5-3 \mathrm{~cm}$ long. Leaf blade $0.5-0.7 \mathrm{~cm}$ broad. Leaf veins not prominent. Leaf veins not black. Leaf margin entire. Leaf margin flat. Leaf apex obtuse. Leaf surface smooth. Leaves white-tomentose. Leaf venation pinnate. Inflorescence lax verticillasters. Flowers pedicelled. Calyx nearly regular. Calyx not funnel-shaped. Calyx veins not prominent. Calyx teeth 5 or less. Calyx teeth not hooked. Calyx teeth not spiny-tipped. Posterior sepal 5-8 $\mathrm{mm}$ long. Veins in calyx tube 15 . Outer surface of petal lobes hairy. Upper lip of corolla flatdeflexed. Corolla tube included in calyx. Stamens 4. Stamens included. Staminal filaments hairy. Filament-connective attachment not lever-like. Ovary glabrous. Style glabrous. Style gynobasic. Stigma 2 elongate arms. Stigmatic lobes equal. Fruit 4 nutlets. Eglandular hairs on leaves absent. Sessile glandular hairs on leaves absent. Stalked glandular hairs on leaves absent. Branched hairs on leaves present. Sessile glandular hairs on calyx present. Stalked glandular hairs on calyx absent. Branched hairs on calyx present.

Teucrium brevifolium Schreb. Plant woody. Stem hairy. Stem terete. Stem prostrate. Stolons absent. Leaves sessile-subsessile. Leaves simple. Leaf blade linear-lanceolate. Leaf blade $0.7-0.8 \mathrm{~cm}$ long. Leaf blade $0.15 \mathrm{~cm}$ broad. Leaf veins prominent. Leaf veins not black. Leaf margin entire. Leaf margin flat. Leaf apex obtuse. Leaf surface smooth. Leaves white-tomentose. Leaf uninerved. Inflorescence terminal globose aggregate. Flowers sessile. Calyx nearly regular. Calyx not funnel-shaped. Calyx veins not prominent. Calyx teeth 5 or less. Calyx teeth not hooked. Calyx teeth not spiny-tipped. Posterior sepal 3-5 mm long. Veins in calyx tube 10 . Outer surface of petal lobes glabrous. Upper lip of corolla reduced. Corolla tube included in calyx. Stamens 4. Stamens exserted. Staminal filaments glabrous. Filament-connective attachment not lever-like. Ovary glabrous. Style glabrous. Style terminal. Stigma 2 elongate arms. Stigmatic lobes unequal. Fruit drupe. Eglandular hairs on leaves present. Sessile glandular hairs on leaves absent. Stalked glandular hairs on leaves absent. Branched hairs on leaves absent. Sessile glandular hairs on calyx absent. Stalked glandular hairs on calyx absent. Branched hairs on calyx absent.

Teucrium decaisnei C. Presl Plant woody. Stem hairy. Stem terete. Stem erect. Stolons absent. Leaves sessile-subsessile. Leaves simple. Leaf blade linear-lanceolate. Leaf blade $0.5-$ $1.2 \mathrm{~cm}$ long. Leaf blade $0.2-0.3 \mathrm{~cm}$ broad. Leaf veins not prominent. Leaf veins not black. Leaf margin denticulate-serrate. Leaf margin flat. Leaf apex obtuse. Leaf surface smooth. Leaves whitetomentose. Leaf venation pinnate. Inflorescence terminal globose aggregate. Flowers sessile. Calyx nearly regular. Calyx not funnel-shaped. Calyx veins not prominent. Calyx teeth 5 or less. Calyx teeth not hooked. Calyx teeth not spiny-tipped. Posterior sepal $1.5-3 \mathrm{~mm}$ long. Veins in calyx tube 10. Outer surface of petal lobes glabrous. Upper lip of corolla reduced. Corolla tube included in calyx. Stamens 4. Stamens exserted. Staminal filaments glabrous. Filament-connective attachment not lever-like. Ovary glabrous. Style glabrous. Style terminal. Stigma 2 elongate arms. Stigmatic lobes unequal. Fruit drupe. Eglandular hairs on leaves present. Sessile glandular hairs on leaves present. Stalked glandular hairs on leaves absent. Branched hairs on leaves absent. Sessile glandular hairs on calyx absent. Stalked glandular hairs on calyx absent. Branched hairs on calyx absent.

Teucrium leucocladum Boiss. Plant woody. 
Stem hairy. Stem terete. Stem erect. Stolons absent. Leaves sessile-subsessile. Leaves simple. Leaf blade linear-lanceolate. Leaf blade $0.9-1.1 \mathrm{~cm}$ long. Leaf blade $0.2-0.4 \mathrm{~cm}$ broad. Leaf veins not prominent. Leaf veins not black. Leaf margin sinuate. Leaf margin flat. Leaf apex obtuse. Leaf surface rugose. Leaves not whitetomentose. Leaf uninerved. Inflorescence terminal globose aggregate. Flowers sessile. Calyx nearly regular. Calyx not funnel-shaped. Calyx veins not prominent. Calyx teeth 5 or less. Calyx teeth not hooked. Calyx teeth not spiny-tipped. Posterior sepal 2-4 mm long. Veins in calyx tube 10 . Outer surface of petal lobes hairy. Upper lip of corolla reduced. Corolla tube included in calyx. Stamens 4. Stamens exserted. Staminal filaments glabrous. Filament-connective attachment not lever-like. Ovary glabrous. Style glabrous. Style terminal. Stigma 2 elongate arms. Stigmatic lobes unequal. Fruit drupe. Eglandular hairs on leaves present. Sessile glandular hairs on leaves absent. Stalked glandular hairs on leaves absent. Branched hairs on leaves absent. Sessile glandular hairs on calyx absent. Stalked glandular hairs on calyx absent. Branched hairs on calyx absent.

Teucrium polium L. Plant herbaceous. Stem hairy. Stem terete. Stem erect. Stolons absent. Leaves sessile-subsessile. Leaves simple. Leaf blade linear-lanceolate. Leaf blade $1.8 \mathrm{~cm}$ long. Leaf blade $0.4-0.5 \mathrm{~cm}$ broad. Leaf veins not prominent. Leaf veins not black. Leaf margin sinuate. Leaf margin flat. Leaf apex obtuse. Leaf surface rugose. Leaves not white-tomentose. Leaf uninerved. Inflorescence terminal globose aggregate. Flowers sessile. Calyx nearly regular. Calyx not funnel-shaped. Calyx veins not prominent. Calyx teeth 5 or less. Calyx teeth not hooked. Calyx teeth not spiny-tipped. Posterior sepal 2-4 mm long. Veins in calyx tube 10. Outer surface of petal lobes glabrous. Upper lip of corolla reduced. Corolla tube included in calyx. Stamens 4. Stamens included. Staminal filaments glabrous. Filament-connective attachment not lever-like. Ovary glabrous. Style glabrous. Style terminal. Stigma 2 elongate arms. Stigmatic lobes unequal. Fruit drupe. Eglandular hairs on leaves present. Sessile glandular hairs on leaves absent. Stalked glandular hairs on leaves absent. Branched hairs on leaves present. Sessile glandular hairs on calyx absent. Stalked glandular hairs on calyx absent. Branched hairs on calyx present.

Thymbra capitata (L.) Cav. Plant woody. Stem hairy. Stem square. Stem erect. Stolons absent. Leaves sessile-subsessile. Leaves simple. Leaf blade linear-lanceolate. Leaf blade $0.4-0.8 \mathrm{~cm}$ long. Leaf blade $0.1-0.2 \mathrm{~cm}$ broad. Leaf veins not prominent. Leaf veins not black. Leaf margin entire. Leaf margin flat. Leaf apex obtuse. Leaf surface smooth. Leaves white-tomentose. Leaf venation pinnate. Inflorescence terminal globose aggregate. Flowers sessile. Calyx bilabiate. Calyx funnel-shaped. Calyx veins not prominent. Calyx teeth 5 or less. Calyx teeth not hooked. Calyx teeth not spiny-tipped. Posterior sepal 3-5 $\mathrm{mm}$ long. Veins in calyx tube 10 . Outer surface of petal lobes hairy. Upper lip of corolla flatdeflexed. Corolla tube included in calyx. Stamens 4. Stamens exserted. Staminal filaments glabrous. Filament-connective attachment not lever-like. Ovary glabrous. Style glabrous. Style gynobasic. Stigma 2 elongate arms. Stigmatic lobes unequal. Fruit 4 nutlets. Eglandular hairs on leaves present. Sessile glandular hairs on leaves present. Stalked glandular hairs on leaves absent. Branched hairs on leaves absent. Sessile glandular hairs on calyx present. Stalked glandular hairs on calyx absent. Branched hairs on calyx present.

Thymus bovei Benth. Plant woody. Stem hairy. Stem square. Stem erect. Stolons absent. Leaves sessile-subsessile. Leaves simple. Leaf blade linear-lanceolate. Leaf blade $1-1.2 \mathrm{~cm}$ long. Leaf blade $0.2-0.3 \mathrm{~cm}$ broad. Leaf veins not prominent. Leaf veins not black. Leaf margin entire. Leaf margin flat. Leaf apex acute. Leaf surface smooth. Leaves not white-tomentose. Leaf venation pinnate. Inflorescence terminal globose aggregate. Flowers sessile. Calyx bilabiate. Calyx not funnel-shaped. Calyx veins not prominent. Calyx teeth 5 or less. Calyx teeth not hooked. Calyx teeth not spiny-tipped. Posterior sepal 2-4 $\mathrm{mm}$ long. Veins in calyx tube 10 . Outer surface of petal lobes hairy. Upper lip of corolla flatdeflexed. Corolla tube included in calyx. Stamens 4. Stamens included. Staminal filaments glabrous. Filament-connective attachment not lever-like. Ovary glabrous. Style glabrous. Style gynobasic. Stigma capitate. Stigmatic lobes equal. Fruit 4 nutlets. Eglandular hairs on leaves absent. Sessile glandular hairs on leaves absent. Stalked glandular hairs on leaves absent. Branched hairs on leaves absent. Sessile glandular hairs on calyx present. Stalked glandular hairs on calyx absent. Branched hairs on calyx absent.

Thymus decussatus Benth. Plant woody. Stem 
hairy. Stem square. Stem erect. Stolons absent. Leaves sessile-subsessile. Leaves simple. Leaf blade linear-lanceolate. Leaf blade $0.2-0.6 \mathrm{~cm}$ long. Leaf blade $0.1 \mathrm{~cm}$ broad. Leaf veins not prominent. Leaf veins not black. Leaf margin entire. Leaf margin flat. Leaf apex acute. Leaf surface smooth. Leaves white-tomentose. Leaf uninerved. Inflorescence terminal globose aggregate. Flowers sessile. Calyx nearly regular. Calyx funnel-shaped. Calyx veins prominent. Calyx teeth 5 or less. Calyx teeth not hooked. Calyx teeth not spiny-tipped. Posterior sepal 3-4 $\mathrm{mm}$ long. Veins in calyx tube 10 . Outer surface of petal lobes hairy. Upper lip of corolla flatdeflexed. Corolla tube included in calyx. Stamens 4. Stamens exserted. Staminal filaments glabrous. Filament-connective attachment not lever-like. Ovary glabrous. Style glabrous. Style gynobasic. Stigma capitate. Stigmatic lobes equal. Fruit 4 nutlets. Eglandular hairs on leaves present. Sessile glandular hairs on leaves present. Stalked glandular hairs on leaves absent. Branched hairs on leaves absent. Sessile glandular hairs on calyx present. Stalked glandular hairs on calyx absent. Branched hairs on calyx absent.

Ziziphora capitata L. Plant herbaceous. Stem hairy. Stem square. Stem erect. Stolons absent. Leaves petioled. Leaves simple. Leaf blade oblong-ovate. Leaf blade $2.4-2.6 \mathrm{~cm}$ long. Leaf blade $1-1.2 \mathrm{~cm}$ broad. Leaf veins prominent. Leaf veins not black. Leaf margin entire. Leaf margin flat. Leaf apex acute. Leaf surface smooth. Leaves white-tomentose. Leaf venation pinnate. Inflorescence terminal globose aggregate. Flowers pedicelled. Calyx bilabiate. Calyx not funnelshaped. Calyx veins not prominent. Calyx teeth 5 or less. Calyx teeth not hooked. Calyx teeth not spiny-tipped. Posterior sepal 6-9 $\mathrm{mm}$ long. Veins in calyx tube 13 . Outer surface of petal lobes glabrous. Upper lip of corolla flat-deflexed. Corolla tube much longer than calyx. Stamens 2. Stamens included. Staminal filaments glabrous. Filament-connective attachment not lever-like. Ovary glabrous. Style glabrous. Style gynobasic. Stigma 2 elongate arms. Stigmatic lobes unequal. Fruit 4 nutlets. Eglandular hairs on leaves present. Sessile glandular hairs on leaves absent. Stalked glandular hairs on leaves present. Branched hairs on leaves absent. Sessile glandular hairs on calyx absent. Stalked glandular hairs on calyx absent. Branched hairs on calyx present.

Ziziphora tenuior L. Plant herbaceous. Stem hairy. Stem square. Stem erect. Stolons absent. Leaves sessile-subsessile. Leaves simple. Leaf blade linear-lanceolate. Leaf blade $1.7-1.9 \mathrm{~cm}$ long. Leaf blade $0.2-0.3 \mathrm{~cm}$ broad. Leaf veins not prominent. Leaf veins not black. Leaf margin entire. Leaf margin flat. Leaf apex acute. Leaf surface rugose. Leaves not white-tomentose. Leaf venation pinnate. Inflorescence dense spikelike. Flowers sessile. Calyx bilabiate. Calyx not funnel-shaped. Calyx veins not prominent. Calyx teeth 5 or less. Calyx teeth not hooked. Calyx teeth not spiny-tipped. Posterior sepal 5-8 mm long. Veins in calyx tube 13 . Outer surface of petal lobes glabrous. Upper lip of corolla flat-deflexed. Corolla tube much longer than calyx. Stamens 2. Stamens included. Staminal filaments glabrous. Filament-connective attachment not lever-like. Ovary glabrous. Style glabrous. Style gynobasic. Stigma 2 elongate arms. Stigmatic lobes unequal. Fruit 4 nutlets. Eglandular hairs on leaves present. Sessile glandular hairs on leaves present. Stalked glandular hairs on leaves absent. Branched hairs on leaves absent. Sessile glandular hairs on calyx present. Stalked glandular hairs on calyx absent. Branched hairs on calyx absent.

\section{Discussion}

The present key is based on a much wider range of characters from vegetative and floral morphology than in any previous floristic treatment. The characters were recorded and used in key generation in a strictly comparative way, so that alternative entries of any couplet in the key are distinguished from each other by means of contrasting character-states of the same character or set of characters. The prelude to the key shows that of the 48 characters recorded for each of the 54 taxa, only 37 were sufficient to generate the key successfully with a surplus of 11 characters. All 48 characters are included in the detailed description of every taxon, thus serving the allimportant function of confirming the identity of these taxa.

Comparison between the DELTA-generated key to the Lamiaceae and its manually constructed counterparts shows that the former is a clear improvement over the latter. Among the numerous advantages of the key provided in the present study: (i) Instead of the user having to go through a manually constructed key to the genera of a given family and then through another key to the species and infra-specific taxa of a given genus, 
the computer-generated key is compact and leads directly to the full scientific names of plants and their respective author citations, (ii) Character definition can be manipulated easily to ensure that they are clearly and accurately observable with the maximum contrast between states of the same character, (iii) The distinction between a pair of alternative entries in the same couplet is strictly comparative, (iv) Most of the couplets in the key are diagnosed by combinations of correlated characters instead of single characters, (v) Character-states separating alternative entries of the same couplet are listed in the same order, thus facilitating the user's task of deciding which entry applies to the unknown plant specimen being identified, and (vi) The flora is so dynamic that some species may disappear and others may invade the country and, unlike the manuallyconstructed fixed keys, the computer-generated keys are equally flexible to cater for such changes.

There are some discrepancies between the taxa representing the Lamiaceae in the present study and those reported by Boulos (2002) in the latest comprehensive account of the flora of Egypt. Instances of these discrepancies are:

(i) Thymus capitatus (L.) Link was always recorded in Egypt by this name (e.g., Täckholm, 1956, 1964 and Boulos, 2000), with Satureja capitata L. as basionym and Coridothymus capitatus (L.) Rchb.f. as synonym, thus omitting the other synonym Thymbra capitata (L.) Cav. But this neglected synonym is shown in the database of the Plant List [http://www.theplantlist.org/] to be the accepted name of this species and we used it in the present study. This is the first representation of the genus Thymbra in Egypt and the number of genera of Lamiaceae reported by Boulos has been consequently increased to 24 .

(ii) The three species Leucas urticifolia (Vahl) R.Br. (from Gebel Elba), Micromeria serbaliana Danin \& Hedge and Origanum isthmicum Danin (both from Sinai) included in Boulos' (2002) flora are not found in the present key to the labiates of Egypt because no specimens of them were located in any of the local herbaria. Flexibility of the DELTA suite of key-generating programs is such that whenever any specimens of these species is re-discovered, they can be added easily to the data matrix and a re-run of the program would update the present key.

\section{$\underline{\text { References }}$}

Balali, P., Soodi, M. and Saeidnia, S. (2012) Protective effects of some medicinal plants from Lamiaceae against beta-amyloid induced toxicity in $\mathrm{PC} 12$ cell. Tehran University Medical Journal, 70(7), 402-409.

Bergianska (2014) [http://angio.bergianska.se/asterids/ Boraginales/Boraginales.html].

Boulos, L. (2002). "Flora of Egypt". Vol. 3. Verbenaceae-Compositae. Al Hadara Publishing, Cairo. 373p.

Caldas, G.F.R., Oliviera, A.R.da S., Araújo, A.V., Quixabeira, D.C.A., Silva-Neto, J. da C., CostaSilva, J.H., de Menezes, I.R.A., Ferreira, F., Leite, A.C.L., da Costa, J.G.M. and Wanderley, A.G. (2014) Gastroprotective and ulcer healing effects of essential oils of Hyptis martiusii Benth. PLOS. [https://doi.org/10.1371/journal.pone.0084400].

Cantino, P.D. (1992) Evidence for a polyphyletic origin of the Labiatae. Annals of the Missouri Botanical Garden, 79, 361-379.

Chase, M.W., Christenhusz, M.J.M., Fay, M.F., Byng, J.W., Judd, W.S., Soltis, D.E., Mabberley, D.J., Sennikov, A.N., Soltis, P.S., Stevens, P.F., Briggs, Barbara, Brockington, S., Chautems, A., Clark, J.C., Conran, J., Haston, Elspeth, Möller, M., Moore, M., Olmstead, R. and Perret, M. (2016) An update of the Angiosperm Phylogeny Group classification for the orders and families of flowering plants. (The angiosperm phylogeny group, APG IV). Botanical Journal of the Linnean Society, 181(1), 1-20.

Dallwitz, M.J., Paine, T.A. and Zurcher, E.J. (2000) User's guide to the DELTA system: A general system for processing taxonomic descriptions. 4.12 ed. CSIRO Division of Entomology, Canberra, ACT, Australia. [also available at: http://delta-intkey.com].

Delnavazi, M.-R., Baba-ali, F., Soufiabadi, S., Sherafatmand, M., Ghahremani, F., Tavakoli, S. and Yassa, N. (2014) Essential oil composition, antioxidant activity and total phenolic content of some Lamiaceae taxa growing in Northwest of 
Iran. Pharmaceutical Sciences, 20(1), 22-28.

El-Gazzar, A.I.H. (1969) A taxonomic study of Labiatae and related genera. Ph.D. Thesis, Southampton University, England. 146p.

El-Gazzar, A. and Watson, L. (1967) Consequences of an escape from floral minutiae and floristics in certain Labiatae. Taxon, 16, 186-189.

El-Gazzar, A. and Watson, L. (1970) A taxonomic study of Labiatae and related genera. The New Phytologist, 69, 451-486.

El-Gazzar, A., Watson, L., Williams, W.T. and Lance, G.N. (1968) The taxonomy of Salvia: a test of two radically different numerical methods. Journal of the Linnaean Society of London (Bot.), 60, 237250 .

El-Gazzar, A., El-Saied, A.S.M., Hammouda, A.A. and Soliman, M.A. (2008 a) Computer-generated keys to the flora of Egypt. 1. The Leguminosae (Mimosoideae and Caesalpinioideae). Taeckholmia, 28, 185-208.

El-Gazzar, A., Rabei, S.H., Aboel Atta, A.I., Kamel, E.A., Loutfy, M.H.A. and Shalabi, L.F. (2008 b) Computer-generated keys to the flora of Egypt. 2. The Chenopodiaceae. Taeckholmia, 28, 209-225.

El-Gazzar, A., Abdel-Ghani, M.M., Aboel Atta, A.I. and Shalabi, L.F.A. (2009 a) Computer-generated keys to the flora of Egypt. 4. The Plantaginaceae. Taeckholmia, 29, 115-131.

El-Gazzar, A., El-Ghareeb, R. and Toto, S.M. (2009 b) Computer-generated Keys to the Flora of Egypt. 3. The Solanaceae. Taeckholmia, 29, 47-73.

El-Gazzar, A., Abdel-Ghani, M.M. and Khattab, A.A. (2012) Computer-generated keys to the flora of Egypt. 5. The Leguminosae-Papilionoideae. Assiut University Journal of Botany, 41(2), 129-181.

El-Gazzar, A., El-Ghamery, A., El-Saied, A., Khattab, A.H. and El-Kady, A.A. (2015 a) Computergenerated keys to the flora of Egypt. 6. The Boraginaceae. Annals of Agricultural Science, 60(1), 67-85.

El-Gazzar, A., Khafagi, A.A., El-Husseini, N. and Mostafa, N.A.M. (2015 b) Computer-generated keys to the flora of Egypt. 7. The Acanthaceae.
Annals of Agricultural Science, 60(2), 257-277.

El-Husseini, N. (1986) A taxonomic revision of Labiatae in Egypt. Ph.D. Thesis. Faculty of Science, Cairo University. 287p.

Guenther, E. (1974) "The Essential Oils, Individual Essential Oils of the Plant Families Rutaceae and Labiatae". Vol. III. R.E. Krieger Publishing Company, New York.

Hagemann, J.M., Earle, F.R., Wolff, I.A. and Barclay, A.S. (1967) Search for new industrial oils. XIV. Seed oils of Labiatae. Lipids, 2, 371-380.

Hegnauer, R. (1966) "Chemotaxonomie der Pflanzenfamilien", Vol. 4. Stuttgart.

Hussain, A.I., Anwar, F., Iqbal, T. and Bhatti, I.A. (2011) Antioxidant attributes of four Lamiaceae essential oils. Pakistan Journal of Botany, 43(2), 1315-1321.

Medjahed, F., Merouane, A., Saadi, A., Bader, A., Cioni, P.L. and Flamini, G. (2016) Chemical profile and antifungal potential of essential oils from leaves and flowers of Salvia algeriensis (Desf.): A comparative study. Chilean Journal of Agricultural Research. [http://dx.doi.org/10.4067/ S0718/].

Mikaili, P., Mojaverrostami, S., Moloudizargari, M. and Aghajanshakeri, S. (2013) Pharmacological and therapeutic effects of Mentha longifolia L. and its main constituent, menthol. Ancient Science of Life, 33(2), 131-138.

Montasir, A.H. and Hassib, M. (1956) "Illustrated Manual Flora of Egypt". Part 1. Dicotyledons. Imprimeri Misr, S.A.E., Cairo. 615p.

Mossa, A.H. (2016). Green pesticides: Essential oils as biopesticides in insect-pest management. Journal of Environmental Science and Technology, 9(5), 354-378.

Muschler, R. (1912) "A Manual Flora of Egypt". 2 Vols. R. Friedlaender \& Sohn, Berlin. 1312p.

Stefanakis, M.K., Touloupakis, E., Anastasopoulos, E., Ghanotakis, D., Katerinopoulos, H.E. and Makridis, P. (2013) Antibacterial activity of essential oils from plants of the genus Origanum. Food Control, 34, 539-546. 
Täckholm, V. (1956) "Students' Flora of Egypt". $1^{\text {st }} \mathrm{ed}$. Anglo-Egyptian Bookshop, Cairo. 649p.

Täckholm, V. (1974) "Students' Flora of Egypt". $2^{\text {nd }}$ ed. Cairo University, Cairo. 888p.

Walker, J.B. and Sytsma, K.J. (2007) Staminal evolution in the genus Salvia: Molecular phylogenetic evidence for multiple origins of stamina lever. Annals of Botany, 100, 375-391.
Watson, L. and Dallwitz, M.J. (1992 onwards) The Families of Flowering Plants: descriptions, identification and information retrieval. Version 19 $9^{\text {th }}$ August 2014. [http://delta-intkey.com].

(Received $15 / 8 / 2018$ accepted $1 / 11 / 2018$ )

\section{بناء مفاتيح لتعريف نباتات القلورة المصرية باستخدام الحاسب الآلي. ^. الفصيلة الشفوية

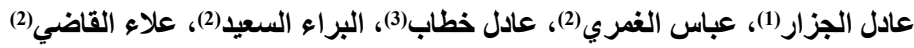

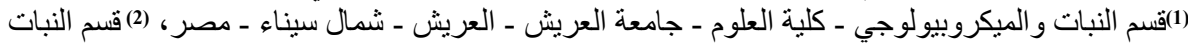

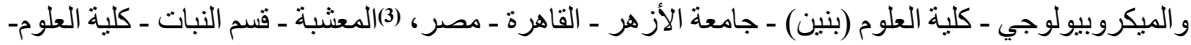

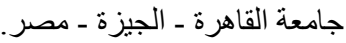

مفاتيح تعريف النباتات في العديد من مجموعات الفلورة المصرية في حاجة ماسة إلى التحسين

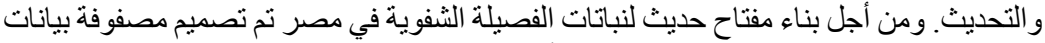

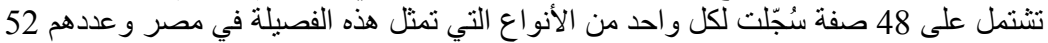

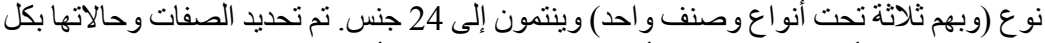

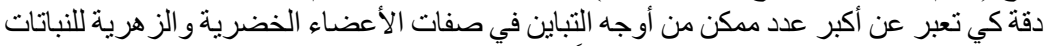

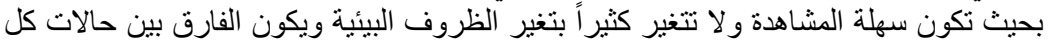

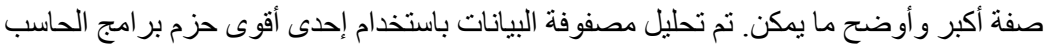

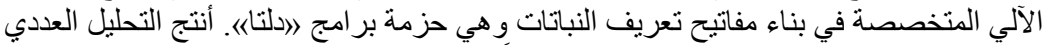

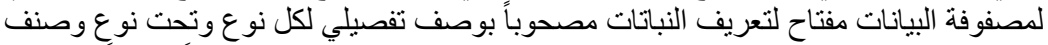

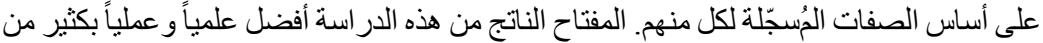

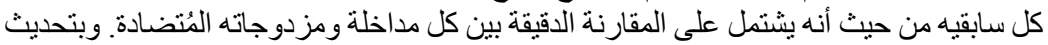

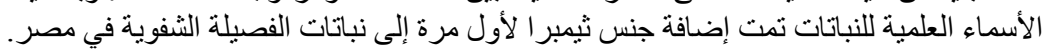

